\title{
Coherent controllers for optical-feedback cooling of quantum oscillators
}

\author{
Ryan Hamerly* and Hideo Mabuchi ${ }^{\dagger}$ \\ Edward L. Ginzton Laboratory, Stanford University, Stanford, California 94305, USA
}

(Received 12 June 2012; published 16 January 2013)

\begin{abstract}
We study the cooling performance of optical-feedback controllers for open optical and mechanical resonators in the linear quadratic Gaussian setting of stochastic control theory. We utilize analysis and numerical optimization of closed-loop models based on quantum stochastic differential equations to show that coherent control schemes, where we embed the resonator in an interferometer to achieve all-optical feedback, can outperform optimal measurement-based feedback control schemes in the quantum regime of low steady-state excitation number. These performance gains are attributed to the coherent controller's ability to simultaneously process both quadratures of an optical probe field without measurement or loss of fidelity, and may guide the design of coherent feedback schemes for more general problems of robust nonlinear and robust control.
\end{abstract}

DOI: 10.1103/PhysRevA.87.013815

PACS number(s): 42.50.Wk, 02.30.Yy, 85.85.+j

\section{INTRODUCTION}

As present-day engineering relies broadly and implicitly on real-time feedback control methodology [1], it is difficult to imagine our nascent explorations of quantum engineering advancing to technological relevance without rigorous extensions of core control theory to incorporate novel features of quantum dynamics, stochastics, and measurement. While significant progress has been made recently in terms of analyzing quantum feedback systems [2-7] and in experimental demonstrations of quantum feedback control [8-17], we still have a relatively limited understanding of systematic approaches to quantum control design and of the qualitative role of quantum coherence and entanglement between the plant and controller in a feedback loop.

Within the elementary context of linear open quantum systems, James, Nurdin, and Petersen [18,19] have utilized interconnection-models-based quantum stochastic differential equations (QSDEs) [20-23] to develop quantum generalizations of the traditional paradigms of $\mathcal{H}^{\infty}$ and linear quadratic Gaussian (LQG) optimal control. While some of the most exciting potential applications of quantum feedback control involve nonlinear dynamics and/or non-Gaussian noises [24-27], the linear setting is an essential starting point for rigorous study and presents crucial advantages in terms of analytic and computational tractability.

Here we focus on a theoretical investigation of steadystate cooling of open quantum oscillators such as optical and optomechanical resonators subject to stationary heating, damping, and optical probing and feedback. We work within an LQG framework as in the recent paper of Nurdin, James, and Petersen [19] and utilize numerical optimization together with fundamental analytic results [1] bounding the best possible LQG performance of measurement-based feedback control

\footnotetext{
*rhamerly@stanford.edu

†hmabuchi@stanford.edu
}

Published by the American Physical Society under the terms of the Creative Commons Attribution 3.0 License. Further distribution of this work must maintain attribution to the author(s) and the published article's title, journal citation, and DOI. schemes to establish and to interpret quantitative advantages of coherent feedback for cooling-type performance metrics in certain parameter regimes.

Following recent convention, as in Refs. [14,18,19], we will here refer to measurement-based controllers as "classical" controllers and to coherent feedback controllers as "quantum" controllers. This terminology reflects the general distinction that the signal processing required to determine LQG-optimal control actions from a real-time measurement signal can be implemented by a classical electric circuit, while all of the hardware in a coherent feedback loop must be physically describable using quantum mechanics (typically with weak damping).

\section{LINEAR SYSTEMS}

Quantum harmonic oscillators can be modeled as cascadable open quantum systems using the SLH framework [28,29] and the associated QSDEs. In the SLH framework, any open quantum system may be described as a triple:

$$
G=(S, L, H)
$$

where $S$ is a scattering matrix, $L$ is a coupling vector, and $H$ is the Hamiltonian operator for the system's internal degrees of freedom. For a linear system with an internal state $x, S_{i j}$ is independent of the internal state, $L_{i}=\Lambda_{i} x+\lambda_{i}$ is at most linear, and $H=\frac{1}{2} x^{\mathrm{T}} R x+r^{\mathrm{T}} x$ is at most quadratic.

Armed with an SLH representation, the most efficient way to simulate a linear quantum system is to solve the QSDEs, which represent coupled Heisenberg equations of motion for system operators and input-output quantum stochastic processes. Following the work of James, Nurdin, and Petersen [18] we write the QSDEs for a linear system in the state-space form,

$$
\begin{aligned}
& d x(t)=[A x(t)+a] d t+B d a(t), \\
& d \tilde{a}(t)=[C x(t)+c] d t+D d a(t) .
\end{aligned}
$$

Here $x(t)$ gives the plant's internal variables; this is a Hermitian, operator-valued vector. $A, B, C$, and $D$ are real matrices; $a$ and $c$ are real vectors. The processes $d a(t)$ and $d \tilde{a}(t)$ are quantum stochastic processes for the inputs and outputs, respectively. For convenience, we make them 
Hermitian as well; for a given port, one has $d a_{i}=\left(d A_{i}+d A_{i}^{\dagger}\right.$, $\left.\left(d A_{i}-d A_{i}^{\dagger}\right) / i\right)$, where $d A(t)$ is the quantum Wiener process [30,31] following the Itô rule $d A_{i} d A_{j}^{\dagger}=\delta_{i j} d t$.

Defining $\Theta_{i j}=\left[x_{i}, x_{j}\right] / 2 i$ as the commutator matrix, the $A B C D$ parameters of Eq. (2) can be related to the SLH parameters as follows:

$$
\begin{aligned}
& A=2 \Theta\left(R+\frac{1}{4} \tilde{\Lambda}^{\mathrm{T}} J \tilde{\Lambda}\right), \quad B=\Theta \tilde{\Lambda}^{\mathrm{T}} J \tilde{S}, \\
& C=\tilde{\Lambda}, \quad D=\tilde{S}, \\
& a=2 \Theta\left(r+\frac{1}{4} \tilde{\Lambda}^{\mathrm{T}} J \tilde{\lambda}\right), \quad c=\tilde{\lambda} .
\end{aligned}
$$

(Here $\tilde{S}, \tilde{\Lambda}$, and $\tilde{\lambda}$ are real matrices that can be easily constructed from $S, \Lambda$, and $\lambda$, which are in general complex. $J$ is a canonical antisymmetric matrix of the appropriate size. See Appendix A.)

To measure the performance of a given controller we need to define a cost function. For example, to minimize the plant's response to a noisy input one could minimize the steady-state expectation value of the excitation number $\left\langle a^{\dagger} a\right\rangle$. With (classical) state feedback and in the absence of exogenous noise, such a quadratic cost function would result in a linear quadratic regulator (LQR) optimal control problem [1], but in our optical feedback scenario with Gaussian input fields (vacuum or thermal noise) this becomes a quantum LQG problem [1,19].

It is straightforward to concatenate and cascade linear systems once we have the $A B C D$ models. We have written software in MATHEMATICA to compute the $A B C D$ matrices for an arbitrary linear quantum system. ${ }^{1}$ This borrows many elements from the MODELICA quantum circuit toolkit of Sarma et al. [32], and is similar to the Quantum Hardware Description Language (QHDL) framework of Tezak et al. [33]. The code computes the LQR cost function as a function of the plant and controller properties, and it would not be difficult to extend it to more general cost functions. Thanks to the linearity of our system, simulation is very fast: the complexity is polynomial in the size of the circuit, not exponential as is usually the case for quantum simulations, and for a simple system, it computes the LQR in well under $50 \mathrm{~ms}$.

Given a particular plant, the code is fast enough to perform a multivariate Newton-Raphson optimization scheme to find the (locally) optimal controller parameters. This is possible regardless of whether the controller has any particular structure-if the controller's structure is left arbitrary, the code can simply optimize with respect to the controller's $A B C D$ matrices, subject to the physical realizability conditions

$$
\begin{array}{r}
A \Theta+\Theta A^{\mathrm{T}}+B J B^{\mathrm{T}}=0, \\
\Theta C^{\mathrm{T}}+B J D^{\mathrm{T}}=0, \\
D J D^{\mathrm{T}}=J
\end{array}
$$

that arise from the fact that time evolution should preserve the commutation relations between system and input and output fields $[18,19]$. Optimizing with respect to an "arbitrary" controller takes longer because there are more free parameters, but the code is fast enough for each Newton step to take no more than $1.5 \mathrm{~ms}$ on a standard laptop.

\footnotetext{
${ }^{1}$ Please contact the authors for distribution of the code.
}

We note that the classical steady-state LQG problem is a convex problem, and the optimal steady-state controller parameters can be derived via solution of algebraic Riccati equations [1]. In the quantum case, no such closed-form solutions are known and the realizability constraints (4) make the landscape for numerical optimization nonconvex [19]. Hence, while we can be sure about the classical optimality of measurement-based controllers for the oscillator cooling scenarios we consider, the coherent controllers we find via numerical optimization are merely local minima and can only be considered as candidates for quantum optimality.

\section{CONTROL OF AN OPTICAL CAVITY}

As a simple example of a quantum "plant" system, consider an optical cavity with a noisy input, Fig. 1 . In the controller's absence, the cavity is driven by two vacuum inputs (mirrors $k_{1}$ and $k_{2}$ ) and one thermal input (mirror $k_{3}$ ). Any noise process that is much broader spectrally than the cavity linewidth can be approximated as a "white noise" thermal input. Without such noise, the cavity's internal mode decays quickly to the ground state. The objective in this control problem is to minimize the effect of the noise on the cavity's internal state-in other words, to minimize the photon number $\left\langle a^{\dagger} a\right\rangle$ of the cavity. We accomplish this by sending output 1 through a control circuit and feeding the result back into input 2. This is an LQG feedback control problem.

Five possible controllers are shown in Fig. 1. The classical controllers work by measuring a quadrature from the cavity's output (or in the heterodyne case, splitting the beam and measuring two different quadratures), and applying a feedback signal based on this measurement and the controller's internal state. The "trivial controller" works by feeding the output directly back into mirror 2 of the plant, perhaps with a phase shift. If the light reflecting off mirror 2 is in phase with the light leaking out of the mirror, the light lost through both mirrors interferes constructively, reducing the control objective $\left\langle a^{\dagger} a\right\rangle$ (see also [26]).

The remaining two controllers shown in the figure are coherent controllers with memory. Unlike the trivial controller, the control signal is a function not only of the input field, but also of the input's history. But unlike the classical controllers,

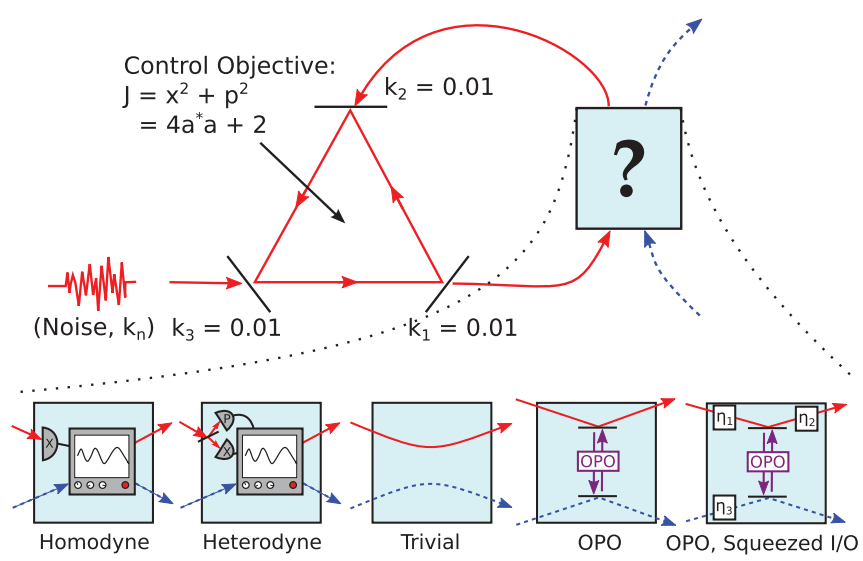

FIG. 1. (Color online) Optical cavity plant system with five possible classical and coherent feedback controllers. 


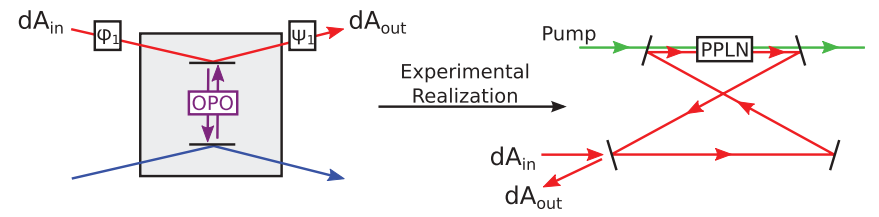

FIG. 2. (Color online) Experimental realization of an OPO with a cavity and a nonlinear crystal.

the input field is not measured; instead, it is coherently processed and the result is fed back into the plant cavity. These designs use an optical parametric oscillator (OPO, as in Fig. 2) to squeeze the optical field.

The OPO will have the following SLH model:

$$
\begin{aligned}
& S=1_{2 \times 2}, \quad L=\left[\sqrt{\kappa_{1}} a, \sqrt{\kappa_{2}} a\right], \\
& H=\frac{1}{4} x^{\mathrm{T}}\left[\begin{array}{cc}
\Delta-\operatorname{Im}(\epsilon) & \operatorname{Re}(\epsilon) \\
\operatorname{Re}(\epsilon) & \Delta+\operatorname{Im}(\epsilon)
\end{array}\right] x .
\end{aligned}
$$

Here, $\kappa_{1}$ is related to the cavity mirror reflectance, and $\kappa_{2}$ to other losses, $\Delta$ is the cavity detuning, and $\epsilon$ is a complex number, whose amplitude encodes the strength of the pump field and the nonlinear medium's $\chi^{(2)}$, and whose phase encodes the pump field's phase [34].

The plant system, an optical cavity with a noisy input, can be modeled as an open quantum system with three couplings, one for each mirror. The SLH model for this system is

$$
S=1_{3 \times 3}, \quad L=\left[\sqrt{k_{1}} a, \sqrt{k_{2}} a, \sqrt{k_{3}} a\right], \quad H=\Delta a^{\dagger} a .
$$

We also need to find the covariance matrix $F_{i j}$ for the noisy inputs $d a_{i}$, defined by $\frac{1}{2}\left\langle d a_{i} d a_{j}+d a_{j} d a_{i}\right\rangle=F_{i j} d t$. Recall that, for vacuum inputs, the fields $d A$ and $d A^{\dagger}$ satisfy the Itô relations $d A d A=d A^{\dagger} d A^{\dagger}=d A^{\dagger} d A=0, d A d A^{\dagger}=d t$ [20,31], leading to the Itô tables in Tables I and II.

For a non-vacuum, thermal input (in this case, the input to mirror $k_{3}$ ), the field $d A$ has additional (unsqueezed) noise, so $d A^{\dagger} d A=k_{n} d t$ for some noise strength $k_{n}>0$, and the rest of the relations are adjusted accordingly, leading to the Itô tables in Tables I and II: In the present system, inputs 1 and 2 are vacuum, and 3 is thermal noise. This gives the following covariance matrix:

$$
F=\left[\begin{array}{cccccc}
1 & 0 & 0 & 0 & 0 & 0 \\
0 & 1 & 0 & 0 & 0 & 0 \\
0 & 0 & 1 & 0 & 0 & 0 \\
0 & 0 & 0 & 1 & 0 & 0 \\
0 & 0 & 0 & 0 & 1+2 k_{n} & 0 \\
0 & 0 & 0 & 0 & 0 & 1+2 k_{n}
\end{array}\right] .
$$

TABLE I. Left: Itô table for the second-order increments $d X d Y$ (with $d X$ in the left margin and $d Y$ in the top margin, given in terms of $d A, d A^{\dagger}$ ), for vacuum noise. Right: Itô table for the increments $d x d y$, with $d x$ and $d y$ given in the left and top margins, in terms of $d a_{x}=d A+d A^{\dagger}, d a_{p}=\left(d A-d A^{\dagger}\right) i$.

\begin{tabular}{l|cc}
$d X / d Y$ & $d A$ & $d A^{\dagger}$ \\
\hline$d A$ & 0 & $d t$
\end{tabular}$\leftrightarrow \quad \leftrightarrow \quad$\begin{tabular}{c|cc}
$d x / d y$ & $d a_{x}$ & $d a_{p}$ \\
\hline$d a_{x}$ & $d t$ & $i d t$ \\
$d a_{p}$ & $-i d t$ & $d t$
\end{tabular}

TABLE II. Itô tables for the second-order increments for thermal noise characterized by noise strength $k_{n}$.

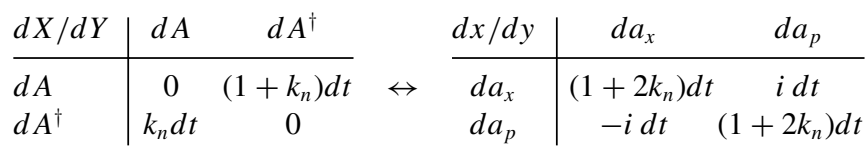

The plant system is easy to set up in our MATHEMATICA package; a sample output is shown in Fig. 3. The package, based on the circuit modeling and analysis framework of Sarma et al. [32], allows one to arbitrarily concatenate and link smaller elements to form larger quantum circuits, as long as all of the components are linear. The feedback control circuit is one example system the package can be used to simulate.

Once the combined plant plus controller system is set up, with its associated $A, B, C$, and $D$ matrices, the covariance matrix $\sigma_{i j}=\frac{1}{2}\left\langle x_{i} x_{j}+x_{j} x_{i}\right\rangle$ can be computed with the Lyapunov equation

$$
A \sigma+\sigma A^{\mathrm{T}}+B F B^{\mathrm{T}}=0 .
$$

For a model system with the parameters

$$
k_{1}=k_{2}=k_{3}=0.01, \quad \Delta=0.1,
$$

we plot the cost function $\left\langle a^{\dagger} a\right\rangle$ as a function of noise $k_{n}$ for the various controller types in Fig. 4. The orange line gives the performance of the plant without a controller. As expected, the photon number rises linearly with the noise power. It is not hard to show that this matches the analytic result

$$
\left\langle a^{\dagger} a\right\rangle_{\mathrm{nc}}=\frac{k_{3}}{k_{1}+k_{2}+k_{3}} k_{n}
$$

that one can derive from the QSDEs.

The trivial controller is simple enough that it also has an analytic solution. The two mirrors, rather than leaking photons separately, do so constructively so that the leakage amplitudes (rather than their powers) add up. This requires the replacement $k_{1}+k_{2} \rightarrow\left(\sqrt{k_{1}}+\sqrt{k_{2}}\right)^{2}$ in Eq. (10), leading to the following result:

$$
\left\langle a^{\dagger} a\right\rangle_{\mathrm{tr}}=\frac{k_{3}}{k_{1}+k_{2}+k_{3}+2 \sqrt{k_{1} k_{2}}} k_{n},
$$

which agrees with the numerical data plotted in Fig. 4.

$$
\begin{aligned}
& \text { Linear SLH Model. } \mathrm{n}_{\text {sys }}=2, \mathrm{n}_{\text {port }}=3 \\
& S=\left(\begin{array}{cccccc}
1 & 0 & 0 & 0 & 0 & 0 \\
0 & 1 & 0 & 0 & 0 & 0 \\
0 & 0 & 1 & 0 & 0 & 0 \\
0 & 0 & 0 & 1 & 0 & 0 \\
0 & 0 & 0 & 0 & 1 & 0 \\
0 & 0 & 0 & 0 & 0 & 1
\end{array}\right), \quad L=\left(\begin{array}{cc}
\sqrt{k_{1}} & 0 \\
0 & \sqrt{k_{1}} \\
\sqrt{k_{2}} & 0 \\
0 & \sqrt{k_{2}} \\
\sqrt{k_{3}} & 0 \\
0 & \sqrt{k_{3}}
\end{array}\right) x, \quad H=\frac{1}{2} x^{T}\left(\begin{array}{cc}
\frac{\Delta}{2} & 0 \\
0 & \frac{\Delta}{2}
\end{array}\right) x \\
& \text { Inputs: } \mathrm{da}_{1} \mathrm{da}_{2} \mathrm{da}_{3} \\
& \text { Outputs: } \mathrm{db}_{1} \quad \mathrm{db}_{2} \quad \mathrm{db}_{3}
\end{aligned}
$$

FIG. 3. Output from our MATHEMATICA package, describing the plant system. 

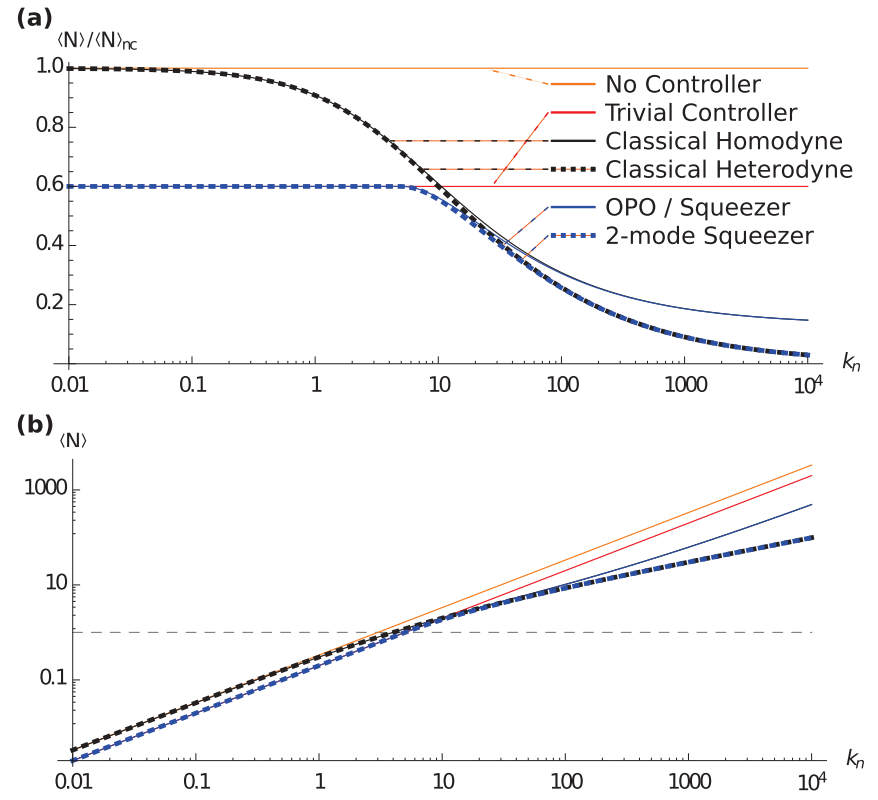

FIG. 4. (Color online) (b) Plant cavity photon number, as a function of noise strength $k_{N}$. The uncontrolled case is shown, as well as the photon number for various control schemes. (a) Photon number relative to the no-control case. Smaller is better.

\section{A. Classical controllers}

More sophisticated are the classical measurement controllers. The first simply makes a homodyne measurement of the $d \tilde{a}_{1 x}$ field. This signal is fed through a classical circuit which generates an output. The heterodyne controller is slightly more complicated, and can be modeled as a two-input homodyne measurement controller in the following circuit (using the notation of Refs. [29,33]; see Appendix A):

$$
(\mathrm{Hom})_{2} \text { in } \triangleleft\left(I_{1} \boxplus e^{i \pi / 2}\right) \triangleleft \mathcal{B}(\alpha) .
$$

In addition to the homodyne controller's parameters, we can also vary the beam-splitter transmittance. (Setting the beamsplitter transmission coefficient $\alpha \rightarrow 1$ would send all the light entering controller input 1 into the $x$-quadrature homodyne detector, so the classical homodyne controller is really a special case of the classical heterodyne controller.)

This example, in particular, illustrates the power of the Gough-James circuit algebra in treating control problems when the controller has a more complex, "circuitlike" structure. Having written code to output the $A B C D$ model for a general $n$-input homodyne controller, it would have been straightforward, albeit tedious, to write additional code for the $n$-input heterodyne controller. But using the Gough-James circuit algebra allows us to write the $n$-input heterodyne system in terms of a $2 n$-input homodyne system, plus some beam splitters and phase shifters, so we get the heterodyne controller for free. By breaking the system into smaller components, we can reduce the total amount of work we need to do in quantum control and simulation problems.

There also exist "analytic" formulas for LQG optimal classical controllers in the classical case. It is not difficult to rewrite Eq. (2) in the standard form for an LQG problem:

$$
\begin{aligned}
& d x=A_{p} x d t+B_{p} d u+d w, \\
& d y=C_{p} x d t+d v .
\end{aligned}
$$

Here $d y$ is the measurement signal, $d u$ is the controller output, and $d w$ and $d v$ are the plant and controller noises, $d w \sim$ $N\left(0, F_{w} d t\right), d v \sim N\left(0, F_{v} d t\right)$. Unfortunately, in this system the noises are correlated; the vacuum noise $d a_{1}$ acts on both the plant and, after reflection off mirror $k_{1}$, the controller. One can define a covariance matrix $M_{i k}=\left\langle d w_{i} d v_{k}\right\rangle$ to account for this correlation.

A common trick is to remove the noise correlations by performing a change of variables [35]. Since $d y-C_{p} x d t-$ $d v=0$, we can subtract this quantity from the first line of Eq. (13) to find an equivalent equation of motion:

$$
\begin{aligned}
d x= & A_{p} x d t+B_{p} d u+d w+M F_{v}^{-1}\left(d y-C_{p} x d t-d v\right) \\
= & \left(A_{p}-M F_{v} C_{p}\right) x+B_{p}\left(d u+B_{p}^{-1} M F_{v}^{-1} d y\right) \\
& +\left(d w-M F_{v}^{-1} d y\right) \\
= & \tilde{A} x+B_{p} d \tilde{u}+d \tilde{w} .
\end{aligned}
$$

Here, the noises $d v$ and $d \tilde{w}$ are uncorrelated. The controller for this plant will consist of a Kalman filter and a feedback:

$$
d \hat{x}=\left(\tilde{A}-B_{p} L-K C_{p}\right) \hat{x}+K d y, \quad d \tilde{u}=-L \hat{x} d t .
$$

The Kalman gain and feedback matrices can be obtained by solving the Riccati equations:

$$
\begin{aligned}
& K=\sigma C^{\mathrm{T}} F_{v}^{-1}\left(A \sigma+\sigma A^{\mathrm{T}}-\sigma C^{\mathrm{T}} F_{v}^{-1} C \sigma=0\right), \\
& L=R^{-1} B^{\mathrm{T}} \lambda\left(A^{\mathrm{T}} \lambda+\lambda A+Q-\lambda B R^{-1} B \lambda=0\right) .
\end{aligned}
$$

(Here $Q$ and $R$ are LQR optimization weights for the plant and controller states; we assume $Q \gg R$.) For this particular case we optimized the classical controllers numerically, but the results agree with the analytical expression. When optimizing the measurement controllers, we found that the best controllers always had dynamics that were much faster than the plant time scales. When this happens, the controller's internal dynamics can be adiabatically eliminated and the controller can be replaced by a simplified "limit model" of the original component [36-38]. When a linear component is adiabatically eliminated, its internal variables are removed and its $A B C D$ model is replaced by the input-output relations

$$
d \tilde{a}=\left(D-C A^{-1} B\right) d a .
$$

The homodyne controller, adiabatically eliminated, becomes

$$
d \tilde{a}_{x}=\xi_{1} d a_{x}+d a_{k 1, x}, \quad d \tilde{a}_{p}=\xi_{2} d a_{x}+d a_{k 1, p} .
$$

In this device, the signal $d a_{x}$ is measured, amplified by factors $c_{1}$ and $c_{2}$, and imprinted onto the output field. The downside of this measurement is the additional noise $d a_{k 1}$ that the output accrues.

The optimal heterodyne controller uses a 50:50 beam splitter so we set $\alpha=1 / \sqrt{2}$ in Eq. (12). It too has very fast 


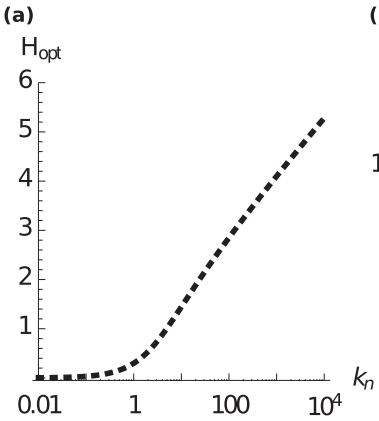

(b)

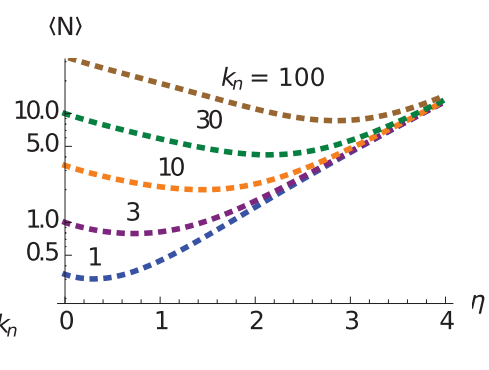

FIG. 5. (Color online) (a) Optimal heterodyne amplification $\eta$, for a classical controller, as a function of plant noise. (b) LQR as a function of controller amplification, for five different noise values.

dynamics that can be adiabatically eliminated to give

$$
\begin{aligned}
d \tilde{a}_{x} & =\xi\left(d a_{x}+d a_{k 1, x}\right)+d a_{k 2, p}, \\
d \tilde{a}_{p} & =\xi\left(d a_{p}-d a_{k 1, p}\right)+d a_{k 2, p},
\end{aligned}
$$

or equivalently

$$
d \tilde{A}=\xi\left(d A+d A_{k 1}^{\dagger}\right)+d A_{k 2} .
$$

The heterodyne controller amplifies both quadratures, but there is an additional noise due to splitting the beam before measurement, $d A_{k 1}$, as well as the measurement noise itself. The LQR can be computed analytically, and the analytic result agrees with the numerical optimizer. Setting $\xi=\sinh (\eta)$, we have

$$
\left\langle a^{\dagger} a\right\rangle_{\mathrm{cl}}=\frac{k_{2} \sinh ^{2} \eta+k_{3} k_{n}}{k_{1}+k_{2}+k_{3}+2 \sqrt{k_{1} k_{2}} \sinh \eta} .
$$

This is plotted in Fig. 5. As the plant noise increases, so does the controller's optimal amplification. It does not do well to increase the amplification indefinitely, however, since this also adds noise into the system. From Fig. 4, one can also see that measurement control does well at reducing the photon number for large $k_{n}$, but in the quantum regime, $k_{n} \lesssim 1$, it has hardly any effect at all.

\section{B. Coherent control}

The three coherent controllers of interest are the cavity controller and the two OPO setups, as shown in Fig. 1. The optimizer consistently showed that the best cavity controller is in fact the trivial controller (which is the special case of a cavity with mirror transmittivity set to zero). Because of this, we do not consider empty cavity controllers in this section. The OPO controllers, on the other hand, have more interesting behavior.

As in the classical case, it was discovered that the best coherent controllers always had dynamics that were much faster than the plant time scales and could be adiabatically eliminated. A single OPO will adiabatically eliminate to a squeezer with the following input-output relations:

$$
d \tilde{a}_{x}=e^{\eta} d a_{x}, \quad d \tilde{a}_{p}=e^{-\eta} d a_{p}
$$

(up to input and output phase shifts). An OPO system with squeezed inputs and outputs, which can in principle replicate any two-port linear quantum system with a single internal

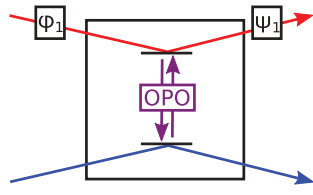

OPO with Phase Shifts

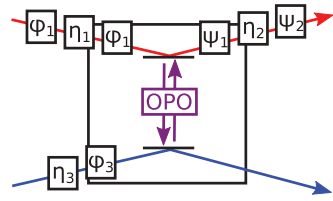

OPO Cavity

Squeezed I/O

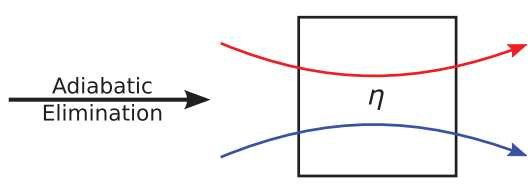

Squeezer

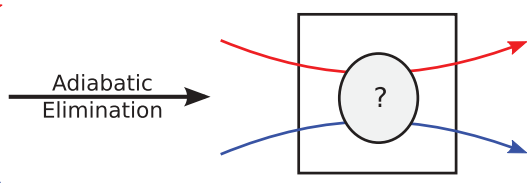

2-mode Squeezing
FIG. 6. (Color online) Optical parametric oscillators adiabatically eliminate into ideal squeezers.

degree of freedom [34], will adiabatically eliminate to arbitrary two-mode squeezer (Fig. 6). As far as this control problem is concerned, the best two-mode squeezer is the linear amplifier, given by the input-output relations

$$
\begin{aligned}
& d \tilde{a}_{1}=\cosh (\eta) d a_{1}+\sinh (\eta) d a_{2}, \\
& d \tilde{a}_{2}=\sinh (\eta) d a_{2}+\cosh (\eta) d a_{2} .
\end{aligned}
$$

Analytic formulas can be derived straightforwardly from the quantum stochastic differential equations. For the squeezer,

$$
\left\langle a^{\dagger} a\right\rangle_{\mathrm{sq}}=\frac{\operatorname{Re}\left[\left(k_{2} \sinh ^{2} \eta+2 k_{n}\right)-2 \frac{k_{2} \sqrt{k_{1} k_{2}} \cosh \eta \sinh ^{2} \eta}{G+2 i \Delta} e^{i \phi}\right]}{\operatorname{Re}\left[G-4 k_{1} k_{2} \sinh ^{2} \eta /(G+2 i \Delta)\right]},
$$

where

$$
G \equiv k_{1}+k_{2}+k_{3}+2 \sqrt{k_{1} k_{2}} \cosh (\eta) e^{i \phi} .
$$

For the linear amplifier,

$$
\left\langle a^{\dagger} a\right\rangle_{2 \mathrm{sq}}=\frac{k_{2} \sinh ^{2} \eta+k_{3} k_{n}}{k_{1}+k_{2}+k_{3}+2 \sqrt{k_{1} k_{2}} \cosh \eta} .
$$

Qualitatively, the results for the heterodyne controller, Eq. (21) and the linear amplifier, Eq. (25) look very similar. Both the heterodyne controller and the linear amplifier reduce the cavity's photon number by amplifying the feedback signal, but also add noise to the system. For equivalent levels of amplification [compare (21), substituting $\sinh \eta \rightarrow \cosh \eta$, to Eq. (25)] the classical controller adds extra noise into the system from the measurement process. When $k_{n}$ and $\eta$ are large, this extra noise is negligible, but in the quantum regime where $k_{n}$ and $\eta$ are $\lesssim 1$, this noise can play a major role in making the linear amplifier outperform the heterodyne controller.

As far as optimization is concerned, Eqs. (24) and (25) are simple enough to apply. Finding the best controller just involves minimizing these functions with respect to $\eta$. But remember that it was not at all obvious that the best quantum controller should be an adiabatically eliminated squeezer. This had to be demonstrated by optimizing the general OPO controller, which has many more parameters, and comparing the result to that of the squeezer. This required a MATHEMATICA 
(a)

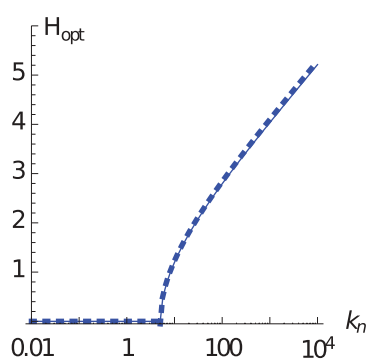

(b)

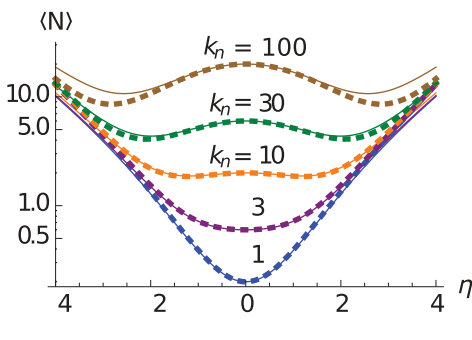

FIG. 7. (Color online) (a) Optimal squeezing for the squeezer (solid) and two-mode squeezer (dotted) controllers. (b) Performance as a function of squeezing for multiple noise levels.

package to quickly convert circuit diagrams to $A B C D$ models, and an efficient optimizer to find the best controller parameters.

Notice that, for large $k_{n}$, the performance of the two quantum controllers follows the classical performance. In the classical limit, the OPO, which reduces to a squeezer, is amplifying a single quadrature and feeding this back into the plant (with the proper phase shift). Likewise, the classical controller measures a single quadrature, amplfies that signal, and sends this back into the plant. Thus, the OPO is a "homodynelike" controller in the classical limit. By contrast, the linear amplifier amplifies both modes equally and feeds back the result, making it a "heterodynelike" controller which tracks the performance of the heterodyne controller in the classical limit.

However, in the quantum regime, this distinction is lost and both of the quantum controllers track the performance of the trivial controller. Below a threshold value of

$$
k_{n, \min }=\frac{k_{1}\left(k_{1}+k_{2}+k_{3}+2 \sqrt{k_{1} k_{2}}\right)}{\sqrt{k_{1} k_{2}} k_{3}}
$$

(for this system, $k_{n, \min }=5$ ), any squeezing will increase the noise in the cavity, so the optimal value of $\eta$ is zero-in other words, for $k_{n} \leqslant 5$, the best controller is the trivial controller.

As Fig. 7 illustrates, when $k_{n}>5$, the best controller has a nonzero amount of squeezing. We plot the controller performance as a function of squeezing for five different noise levels on the right panel of the figure. Intuitively, this is a battle between the noise introduced by squeezing and the noise removed by constructive interference with the light leaking out of mirror 2. When $\eta$ is low, the latter dominates. By increasing the squeezing, we effectively increase the amplitude of the field impinging upon mirror 2. Recall that the trivial controller worked by constructive interference between this field and the light leaking out of mirror 2. By increasing this field's amplitude, we magnify the effect of this interference; this reduces the overall cavity photon number. This explains the $\cosh \eta$ term in the denominator of Eq. (25). But a squeezed vacuum carries photons of its own, and some of these photons leak back into the cavity. If the squeezing is too high, this winds up increasing the photon number, giving rise to the $\sinh ^{2} \eta$ term in Eq. (25). Above the threshold temperature $k_{n \text {,min }}$, the ideal $\eta$ lies somewhere between these extremes.

Below the threshold temperature, the cavity photon number is so low that the interference effect never wins out—squeezing the control field always introduces more photons in the cavity, and the best controller involves looping the output from mirror 1 into mirror 2 without squeezing — the trivial controller.

\section{OPTICAL FEEDBACK CONTROL OF A MECHANICAL OSCILLATOR}

Optomechanical oscillators-mechanical springs that couple to an optical field via a cavity - have been a topic of tremendous recent interest in the physics community [39]. A central goal has been to find ways to exploit optomechanical coupling to cool the mechanical oscillator from ambient temperature to its ground state, using optical feedback.

In this section we analyze the optomechanical oscillator as a coherent control system, with the spring comprising the plant, and with optical probing and feedback. We optimally cool the oscillator by solving the LQG control problem for the cost function $\left\langle b^{\dagger} b\right\rangle$, where $b$ is the spring's annihilation operator. While the control setups we consider may appear impractical from an experimental perspective, we will discuss how they can be related to systems that are more realistic to implement.

At the heart of this control problem is the "adiabatically eliminated cavity," depicted in Fig. 8. If we go into the rotating frame for the light, this has the $S L H$ model

$S=1_{2 \times 2}, \quad L=[\sqrt{\kappa} a, \sqrt{\Omega / Q} b], \quad H=\hbar \Omega b^{\dagger} b+\eta a^{\dagger} a x_{m}$,

where $\Omega$ is the natural spring frequency, $Q$ is the $Q$ factor, $\kappa$ is the cavity decay parameter, and $m$ is the mirror mass. See Table III.

System (27) is nonlinear by virtue of the interaction term $\eta a^{\dagger} a X$. This term is due to the photon pressure of the field in a cavity, which exerts a physical force on the mirror. In the limit that the light mode $a$ evolves much faster than the mechanical mode $b$, we can adiabatically eliminate the former to give an $S L H$ system of the form

$S=\left[\begin{array}{cc}e^{i \phi\left(x_{m}-x_{m 0} ; \eta / \kappa\right)} & 0 \\ 0 & 1\end{array}\right], \quad L=[0, \sqrt{\Omega / Q} b], \quad H=\Omega b^{\dagger} b$,

where

$$
\phi(z ; \eta / \kappa)=2 \tan ^{-1}(2 \eta z / \kappa)
$$

is the phase shift of the cavity reflected light, as a function of the mirror position (we have absorbed a factor -1 in $S$ for convenience). This is still a highly nonlinear system. A real optomechanical oscillator is usually driven by a coherent field, and the output that is measured is generally interfered with an equal and opposite field, so as to discern the phase fluctuations

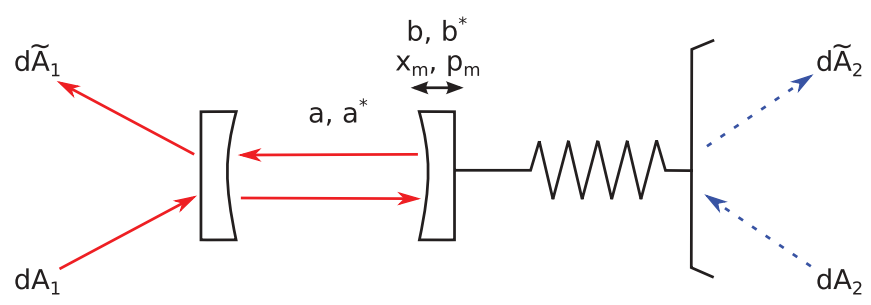

FIG. 8. (Color online) Single cavity with modes $a, a^{\dagger}$, coupled to a mechanical oscillator with modes $b, b^{\dagger}$. 
TABLE III. Parameters for the optical cavity-controller problem. See, e.g., [39].

\begin{tabular}{l|ll|l|l}
\hline \hline Quantity & Value & Quantity & \multicolumn{1}{c}{ Description } & Typical values \\
\hline$K_{i}$ & $=4 \eta r_{i} / \kappa_{i}$ & $P_{i}$ & Laser power in coherent displacement $r_{i}, i=1,2$ & $1 \mu \mathrm{W}-1 \mathrm{~mW}$ \\
$r_{i}$ & $=\sqrt{P_{i} / \hbar \omega}$ & $t_{i}$ & Power transmittance for cavity mirror $i$. Inversely proportional to finesse. & $10^{-6}-10^{-3}$ \\
$\kappa_{i}$ & $=t_{i} c / 2 l_{i}$ & $l_{i}$ & Length of cavity $i$ & $10^{-6}-10^{-1} \mathrm{~m}$ \\
$\eta$ & $=\left(\omega / l_{i}\right) \sqrt{\hbar / 2 m \Omega}$ & $m$ & Mass of spring-mounted mirror & $10^{-15}-10^{-10} \mathrm{~kg}$ \\
$k_{n}$ & $=\left(1-e^{-\hbar \Omega / k T}\right)^{-1}$ & $\Omega$ & Spring oscillation frequency & $\mathrm{kHz}-\mathrm{GHz}$ \\
$k_{m}$ & $=\Omega / Q$ & $Q$ & Spring quality factor & $10^{3}-10^{7}$ \\
& & $\omega$ & Laser frequency & $(2-4) \times 10^{15} / \mathrm{s}$ \\
\hline \hline
\end{tabular}

on a homodyne detector. Thus, the real plant system we are interested in is the adiabatically eliminated cavity sandwiched between two coherent displacements. For a cavity subject to a coherent input of amplitude $r_{1}$, we write this as

$$
\left(\mathrm{Cav}_{1}\right)=L(-r) \triangleleft(\mathrm{Cav}) \triangleleft L(r) .
$$

This has the simple, linear SLH model

$$
S_{1}=1_{2 \times 2}, \quad L_{1}=\left[K_{1} x_{m}, \sqrt{\Omega / Q} b\right], \quad H_{1}=\Omega b^{\dagger} b
$$

with $K_{1}=4 \eta r_{1} / \kappa_{1}$ the effective coupling between the spring and the field, which need not be positive or even real. The $x_{m}$ coupling to the field $d a_{1}$ gives rise to the following input-output relations:

$$
\begin{aligned}
\left(d x_{m}\right)_{1} & =0, \quad\left(d p_{m}\right)_{1}=-2 K_{1} d a_{1 p}, \\
d \tilde{a}_{1 x} & =d a_{1 x}+2 K_{1} x_{m} d t, \quad d \tilde{a}_{1 p}=d a_{1 p} .
\end{aligned}
$$

The state variable $x_{m}$ is imprinted on the output $d \tilde{a}_{1 x}$, so by measuring the $x$ quadrature of the output field, we can deduce the value of $x_{m}$; this allows us to use the mirror as a "measurement" device, learning information from the output field. Note that this works only for $d \tilde{a}_{1 x}$; no information is imprinted onto the $p$ quadrature of the output. Conversely, by sending in a particular input $d a_{1 p}$, we can alter the state of the system; this allows us to use the mirror as a "feedback" device. Note likewise that feedback is not possible via the $d a_{1 x}$ channel, which does not affect the system.

\section{A. Plant system}

The plant-controller setup is shown in Fig. 9. The plant system consists of two (adiabatically eliminated) cavities coupled to the same mirror. The output from the first cavity, $d \tilde{A}_{1}$, goes into the controller, and the controller output is fed back into the second cavity input $d A_{2}$. Not shown are the two coherent displacements (lasers) putting fields $d A_{1}$ and $d A_{k}$ into nonvacuum coherent states. These coherent fields allow us to replace the cavity with model (28) with the linearized model (D5). Since the system is now linear, this becomes an LQG control problem. The combined plant-controller system can be viewed as a feedback loop from output $d \tilde{A}_{1}$ to input $d A_{2}$, or conversely, we can write it as a series product

$$
(\mathrm{Sys})=\left[\left(\mathrm{Cav}_{2} \boxplus I_{1}\right) \triangleleft \mathcal{K} \triangleleft\left(\mathrm{Cav}_{1} \boxplus I_{1}\right)\right] \boxplus(\mathrm{Spr}),
$$

where (Sys) is the combined system, $\mathrm{Cav}_{i}$ is the $i$ th cavity, with the $S L H$ model $\left(1, \sqrt{k_{i}} x_{m},{ }_{-}\right)$, (Spr) gives the spring and phonon couplings, with the $S L H$ model $\left(1, \sqrt{k_{m}} x_{m}, \Omega b^{\dagger} b\right)$, and $\mathcal{K}$ is the controller. See Fig. 10.
The controllers we consider here are not unlike those for the simple cavity. It is not difficult to show using Eq. (33) that the trivial controller amounts to no control at all at best, and additional noise at worst. The classical controller measures the output from mirror cavity 1 and sends an input in to cavity 2 , as a function of the controller's internal state. (Note that we need to consider only a classical controller that measures the $x$ quadrature $d \tilde{a}_{1 x} ; d \tilde{a}_{1 p}$ contains no information about the plant's state.) The simple cavity and OPO cavity coherently process the signal rather than destroying it in a measurement. Finally, we considered the most general coherent controller, an open quantum system specified by arbitrary $A, B, C, D$ matrices satisfying the realizability relations. For the LQG problem of minimizing $\left\langle b^{\dagger} b\right\rangle$, we found optimal controllers in each class for the following plant system:

$$
\begin{aligned}
& \Omega=100 \text { (arbitrary units), } k_{m}=0.01, \\
& Q=10000, \quad k_{n}=10^{-9}-10^{9} .
\end{aligned}
$$

In the optimization, we are allowed to vary both the controller parameters and the couplings $K_{1}, K_{2}$ to the cavities in Eq. (D5). This is because the couplings depend on the input laser powers $P_{i}$ (in addition to the mirror transmittances $t_{i}$ ), which are

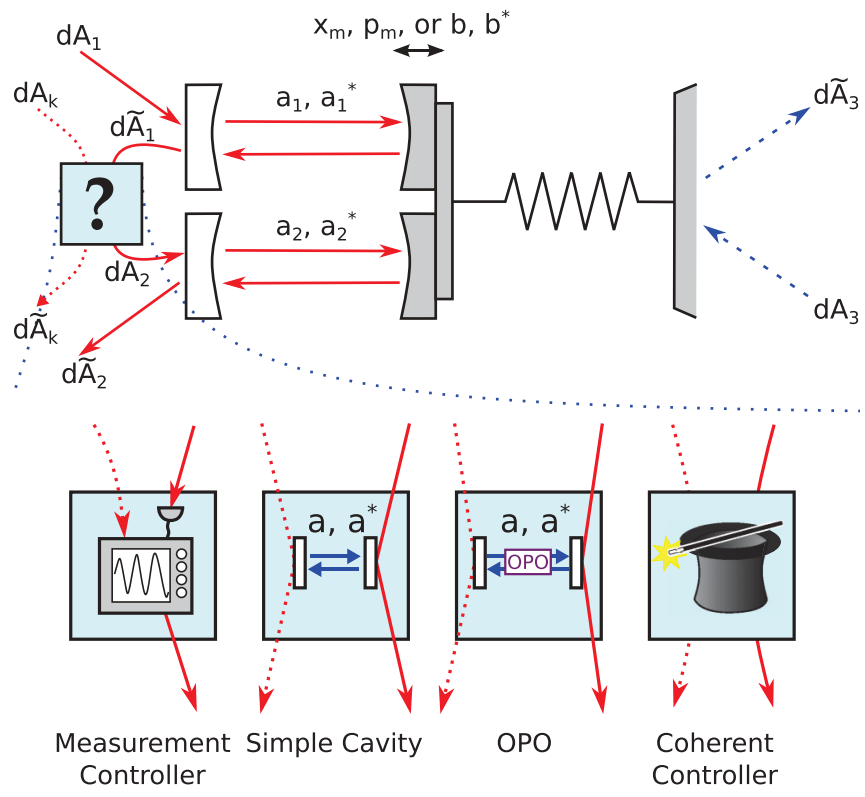

FIG. 9. (Color online) Control-system setup for the mechanicaloscillator cooling problem. Four potential controller designs. 


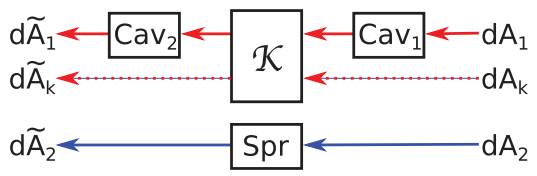

FIG. 10. (Color online) Equivalent view of the plant-controller setup shown in Fig. 9. See Eq. (33).

external quantities (see Table III) rather than fixed properties of the plant itself. Here we will operate primarily under the assumption $K_{1}=-K_{2} \equiv K$; this is a reasonable assumption that avoids classical solutions with divergent controller gain, but we also show that the coherent controllers discussed here outperform the best classical controllers even when this assumption is relaxed.

Figure 11 plots the performance of the measurement, simple cavity, and OPO controllers. For very low ambient temperatures where the noise is weak, the plant is nearly in its ground state to begin with, and none of the controllers can reduce its value. This differs from the optical cavity. In the cavity, we used a "trivial controller" to cause the light leaking out of mirror 1 to interfere constructively with the light leaking out of mirror 2 , increasing the net dissipation from $k_{1}+k_{2}$ to $\left(\sqrt{k_{1}}+\sqrt{k_{2}}\right)^{2}$. No such scheme exists in the oscillator because phonons do not "leak out" of the system in the same way that photons leak out of an optical cavity.

At high temperatures, the best classical controller and the OPO controller do equally well, each reducing the phonon number by a factor of exactly $Q=10000$. The cavity controller does reasonably well, reducing the phonon number by a factor of about $0.354 Q=3540$. These results are not very surprising. The high-temperature limit takes our oscillator into the classical regime, where vacuum noise is negligible and no

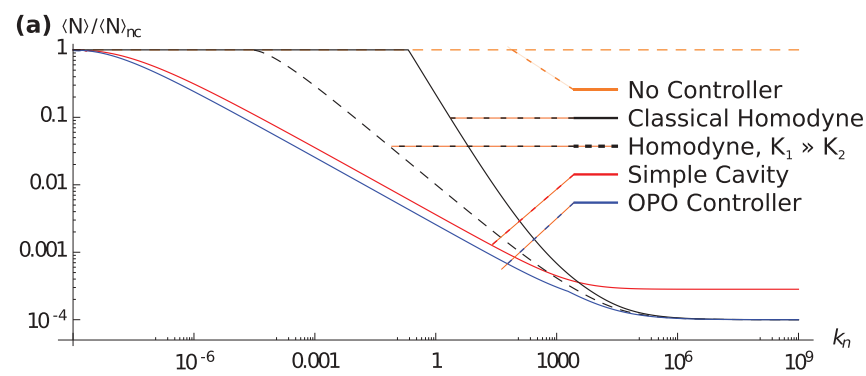

(b)

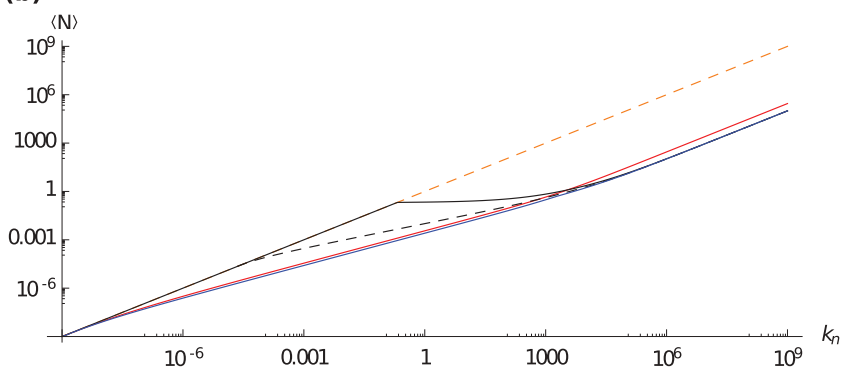

FIG. 11. (Color online) (b) Plot of the average phonon number $\langle N\rangle=\left\langle b^{\dagger} b\right\rangle$ of the mechanical oscillator for three different control schemes. (a) Phonon-number reduction, relative to the no-control case. The general coherent-controller result is not shown, since it overlaps the OPO line, the optimal coherent controller being an OPO cavity. coherent controller can hope to outperform the best classical controller.

The interesting region lies between these two limits. Here, there is a sharp cutoff, near $k_{n} \approx 0.2$, below which the classical measurement controller becomes useless. As explained below, the classical controller must add noise to the system to make a measurement; below a certain threshold, the gains from control are offset by the noise from measurement. In this region, the cavity and OPO controllers do significantly better than the classical controller, in some places by a factor of 100-200.

\section{B. The classical controller}

The classical controller works by measuring the plant output field ( $d A$ in Fig. 12) and inferring the plant's state from this measurement. From the inferred plant state, the controller applies a feedback signal, which is added to an auxiliary vacuum input $d A_{k}$ and sent back to the plant.

The plant output contains two quadratures, but only one of them contains information about the system. Thus, in our classical controller we choose to measure the $x$ quadrature of the output, and necessarily discard the $d A_{p}$. This is the optimal control strategy in the classical case because $d A_{p}$ does not contain any information about the system. Like any LQG optimal controller, the classical controller consists of a Kalman filter, which estimates the plant state, plus a feedback element.

The classical controller adds two sources of noise to the plant. First, by sending a laser through the measurement cavity $\left(\mathrm{Cav}_{1}\right)$, it adds measurement noise, with an amplitude that scales as $O(K)$. Second, the feedback field $d \tilde{A}$ (with a vacuum noise component due to the auxiliary field $d A_{k}$ ) is sent through the controller, adding a feedback noise of equal magnitude, also $O(K)$. Both of these factors increase the cavity phonon number by $O\left(K^{2}\right)$, independent of the noise $k_{n}$. The control loop will decrease the cavity phonon number by an amount proportional to the present phonon number, which increases with $k_{n}$. In the high- $k_{n}$ limit, the "control" term dominates and the coupling $K$ is large. By contrast, in the low- $k_{n}$ limit, the "noise" term is dominant, and the optimal value of $K$ is small or zero-no measurement controller can effectively reduce the phonon number, since the noise incurred will more than offset any gains from control.

An important thing to note is the role the $p$-quadrature field $d a_{p}$ plays in this noise budget. It is true that $d a_{p}$ does not contain any information about the plant state. But this quadrature still plays an important part, since $d a_{p}$ gives rise to the noise in the measurement cavity, and $d \tilde{a}_{p}$ gives rise to the noise in the feedback cavity. Because $d a_{p}$ and
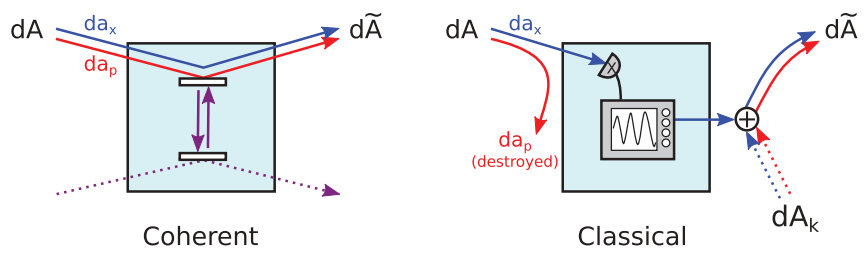

FIG. 12. (Color online) Flow of $x$ - and $p$-quadrature signals (blue and red, respectively) in the classical and coherent controllers. 
$d \tilde{a}_{p}$ are independent (the former being destroyed in the $d a_{x}$ measurement), their noises add up. The beauty of coherent control is that we can process the $x$ field without destroying $d a_{p}$ and the measurement and feedback noises become correlated. If this is done right, they cancel each other out.

If we are free to relax the $K_{1}=-K_{2}$ assumption, then the classical controller does somewhat better (dashed line in Fig. 11), but still underperforms the coherent schemes discussed below. When $K_{1} \neq K_{2}$, the optimal classical controller tends to have $K_{2} \ll K_{1}$, which greatly suppresses the measurement noise. To compensate for this disparity, the controller must have a large classical gain.

It might be thought that a heterodyne-based control scheme like that in Fig. 13 could perform better than the best homodyne controller. After all, the homodyne controller is just a special case of the heterodyne controller, where the beam splitter has a transmissivity of $100 \%$. Moreover, one might imagine using a heterodyne scheme to cycle part of the $d \tilde{a}_{p}$ quadrature back into the plant, canceling out part of the measurement noise with the feedback noise. However, we find numerically that the most general heterodyne controller does not perform any better-either with $K_{1}=K_{2}$ or not. The extra noise added from splitting the beam outweighs any of the benefits of the control scheme.

\section{Simple cavity controller}

An empty optical cavity with two input and output ports has the following SLH model:

$$
S=1_{2 \times 2}, \quad L=\left[\sqrt{\kappa_{1}} a, \sqrt{\kappa_{2}} a\right], \quad H=\Delta a^{\dagger} a .
$$

Here the $\kappa$ 's are mirror decay parameters and $\Delta$ is the detuning of the cavity. The QSDEs for the cavity are easy to derive:

$$
\begin{aligned}
d a & =(-i \Delta-\kappa / 2) a d t+\sqrt{\kappa_{1}} d \tilde{A}_{1}+\sqrt{\kappa_{2}} d \tilde{A}_{2}, \\
d \tilde{A}_{i} & =d A_{i}+\sqrt{\kappa_{1}} a d t .
\end{aligned}
$$

Remember that, in addition to the controller parameters, we can vary the input coherent fields, which allows us to vary the plant's $x_{m}$ coupling $K$. The laser field impinging on cavity 1 adds shot noise to the mirror; in this setup, since $K_{1}=-K_{2} \equiv$ $K$, the shot noise from cavity 1 will exactly cancel the shot noise from cavity 2 (if we let $K_{1}$ and $K_{2}$ vary freely, we find

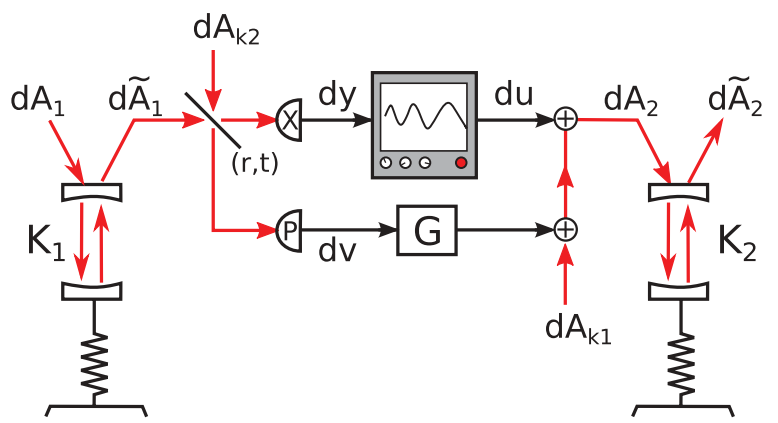

FIG. 13. (Color online) Heterodyne-based measurement controllers, which measure both quadratures of the beam by splitting it, do not outperform the best homodyne controller for this system.

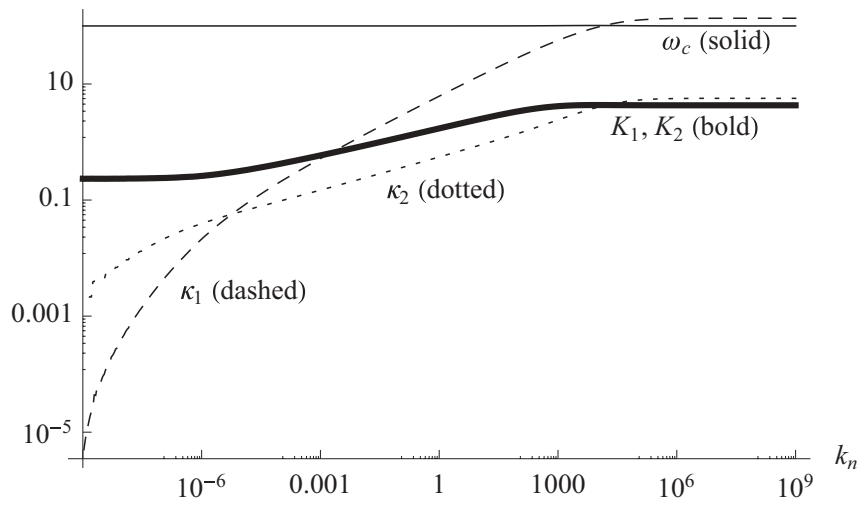

FIG. 14. Parameters of the optimal simple cavity controller, as a function of noise strength.

that the optimal controller has $K_{1}=-K_{2}$ ). As a consequence, the cavity controller has neither measurement nor feedback noise.

The optimal detuning and couplings are plotted in Fig. 14. Not surprisingly, as the noise on the mirror is increased, the couplings $K_{1,2}$ and $\kappa_{1,2}$ increase as well. The detuning $\Delta$, which shows no dependence on the noise power, always remaining at a constant value $\Delta \approx \Omega=100$ for this system, making the cavity-controller setup analogous to two coupled harmonic oscillators, one mechanical and the other optical [40]. Absent the couplings, the quadratures $x=a+a^{\dagger}$, $p=\left(a-a^{\dagger}\right) / i$ would evolve just like the mirror variables $x_{m}, p_{m}$.

This can also be interpreted as a form of sideband cooling. The detuning $\Delta \approx \Omega$ indicates that our control system is being driven by laser light at a frequency $\omega_{\text {cav }}-\Omega$, where $\omega_{\text {cav }}$ is the cavity resonance frequency. The plant-controller coupling serves to convert photons of frequency $\omega_{\text {cav }}-\Omega$ to photons of frequency $\omega_{\text {cav }}$, cooling the oscillator. At high temperatures, we need a large cooling rate to counter the noise; this is achieved by using a cavity with a broad bandwidth $\kappa$, so that both $\omega_{\text {cav }}-\Omega$ and $\omega_{\text {cav }}$ photons interact effectively with the cavity. Conversely, at low temperatures, we need to work in the resolved sideband limit $\kappa \ll \Omega$ to suppress quantum fluctuations of the radiation-pressure force [41-43].

The effects of this cooling are made manifest on the output power spectrum of the photon channel $\tilde{P}_{1}(\omega)=\tilde{A}_{1}(\omega)^{\dagger} \tilde{A}_{1}(\omega)$, where $\tilde{A}_{1}(\omega)$ is the Fourier transform of the stochastic process $d \tilde{A}_{1}(t)$. In the frequency domain, the relevant QSDEs for the combined plant-cavity system are

$$
\begin{aligned}
-i \omega a= & \left\{\left[-i \Delta-\left(\kappa_{1}+\kappa_{2}\right) / 2\right] a+\sqrt{\kappa_{1}} K\left(b+b^{\dagger}\right)\right\} \\
& +i \omega \sqrt{\kappa_{1}} A_{1}+i \omega \sqrt{\kappa_{2}} A_{2}, \\
-i \omega b= & {\left[(-i \Omega-\Omega / 2 Q) b-\sqrt{\kappa_{1}} K\left(a-a^{\dagger}\right)\right] } \\
& +i \omega \sqrt{\Omega / Q} A_{3}, \\
-i \omega \tilde{A}_{1}= & -i \omega A_{1}+\sqrt{\kappa_{1}} a .
\end{aligned}
$$

This power spectrum is plotted in Fig. 15. As the exiting light is blue detuned, it reduces the phonon number in the oscillator, driving it towards the ground state. For small $k_{n}$, when the plant and controller are weakly coupled, there is a single sideband corresponding to the plant's oscillation frequency $\Omega$. When $k_{n}$ is large, the plant and controller become strongly coupled and 


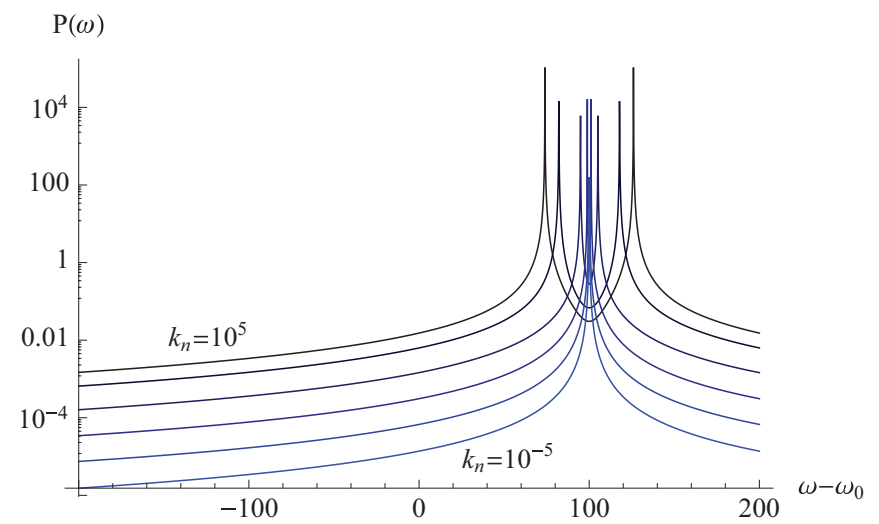

FIG. 15. (Color online) Calculated output spectrum of light exiting the optimal simple cavity controller. Six values of $k_{n}$ are plotted, $10^{5}$ (darkest), $10^{3}, 10^{1}, 10^{-1}, 10^{-3}$, and $10^{-5}$ (lightest).

the combined system resonates at two different frequencies, one larger than $\Omega$ and one smaller. This is the origin of the sideband splitting in the figure.

The system can also be understood as a form of coherent Kalman filtering. Recall that the optimal classical controller works as a Kalman filter, reproducing the state of the plant by measuring one of its outputs. The cost we paid for the Kalman filtering was additional noise added to the system. The cavity controller can also be thought of as a Kalman filter, but one that preserves the coherence of the input signal $d A$. From a quantum mechanical standpoint, in the classical controller, the $p$ quadrature $d a_{p}$ is essentially discarded after the measurement. In the cavity controller, the field retains its coherent properties and the $d a_{p}$ coming out is the same as $d a_{p}$ going in. This makes the noises in the measurement and feedback cavities correlated. In the present setup, they exactly cancel out. This cancellation of the measurement noise is what gives the coherent cavity controller its superior performance, particularly in the low-phonon-number regime.

Measurement sensing experiments [44], particularly in the context of the Laser Interferometer Gravitational-Wave Observatory (LIGO) [45], show similar improvements, but for a different performance metric. This suggests that LQG control is far from the only problem to benefit from this noise cancellation and coherent feedback; similar gains should be expected in all types of control problems when the plant operates in the quantum regime.

\section{OPO cavity controller}

Recall from Eq. (5) that the OPO has the following SLH model:

$$
\begin{aligned}
S & =1_{2 \times 2}, \quad L=\left[\sqrt{\kappa_{1}} a, \sqrt{\kappa_{2}} a\right], \\
H & =\frac{1}{4} x^{\mathrm{T}}\left[\begin{array}{cc}
\Delta-\operatorname{Im}(\epsilon) & \operatorname{Re}(\epsilon) \\
\operatorname{Re}(\epsilon) & \Delta+\operatorname{Im}(\epsilon)
\end{array}\right] x \\
& =\Delta a^{\dagger} a+\frac{\epsilon^{*} a^{2}-\epsilon\left(a^{\dagger}\right)^{2}}{2 i} .
\end{aligned}
$$

For fullest generality, the OPO controller is placed between two phase shifters, so the actual controller is $e^{i \phi_{1}} \triangleleft(\mathrm{OPO}) \triangleleft e^{i \phi_{2}}$. Between the controller, the phase shifters,

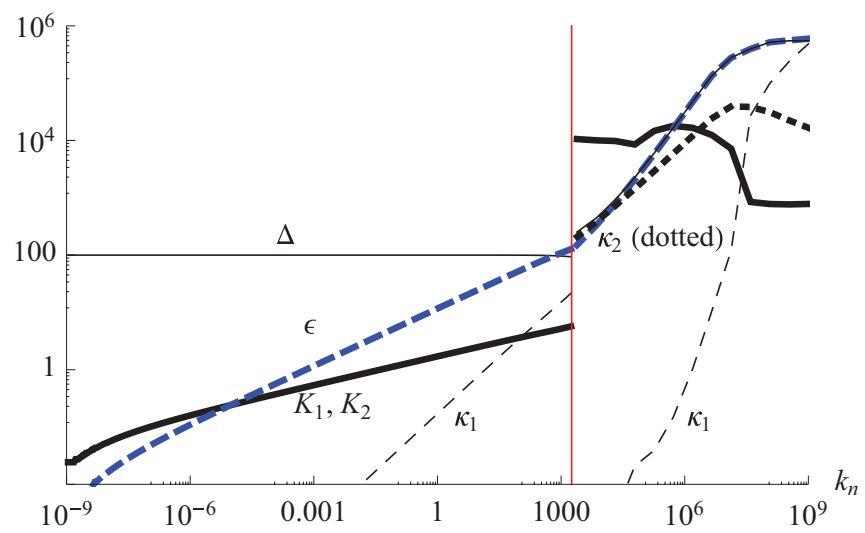

FIG. 16. (Color online) Parameters of the optimal OPO cavity controller, as a function of noise strength.

and the couplings $K_{1,2}$, there are nine free parameters in this LQG problem. The best OPO controller parameters, found using the optimization code, are plotted in Fig. 16. As with the cavity controller, the best OPO controller has $K_{1}=-K_{2}$.

For $k_{n} \lesssim 1800$, the OPO behaves much like the simple cavity. Its detuning is close to $\Omega$, the coupling $K_{1}=-K_{2}$ increases with $k_{n}$, and the mirror losses $\kappa_{1}, \kappa_{2}$, while small, increase with increasing noise $\left(\kappa_{2}\right.$ is too small to be seen on this plot). For the most part, $\epsilon \ll \Delta$ and the OPO squeezing is only a perturbation on the dynamics of an empty cavity.

At $k_{n} \approx 1800$, this changes suddenly. This happens because the OPO controller has two local minima. Below $k_{n} \approx 1800$, the empty-cavity-like local minimum is smaller, but above this threshold, a new minimum dominates. In this regime, the coupling $K$ is much stronger than before and the mirrors $\kappa_{1}, \kappa_{2}$ are much more lossy.

The OPO controller appears to be the best coherent controller one can make for this system. We ran the optimizer for a general coherent controller, subject to no constraints other than the realizability conditions (4). At no point did we find a coherent controller that outperformed the OPO for this system. With this in mind, the discontinuity at $k_{n}$ can be better understood. As the best realizable controller, the OPO must do at least as well as both the simple cavity and the classical controller. For weak noise, the simple cavity outperforms the classical controller, so we expect the OPO to look more like a simple cavity. For strong noise, the classical controller does better, so we expect the OPO to look more like a classical controller, inasmuch as this is possible. There is no reason to assume that the transition between the two must be smooth. It may be marked with bifurcation points, as in Fig. 7 for the cavity control problem, or it may occur with a discontinuity in the parameters. What happens for a general plant plus controller system will depend on the landscape of the cost function, and in particular, the behavior of local minima.

\section{E. More realistic control systems}

The control systems discussed above can be implemented in principle, but they require two separate mirrors and two separate cavities to be coupled to the same mechanical oscillator, which may prove difficult to build in a laboratory. Fortunately, one can show that for the cavity controller and 


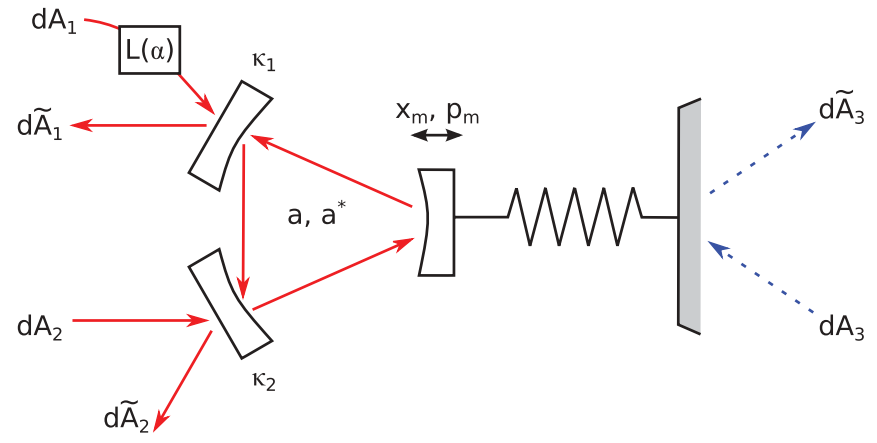

FIG. 17. (Color online) Model for a non-adiabatically-eliminated cavity.

the OPO controller, equivalent systems can be realized using a non-adiabatically-eliminated cavity with one of its mirrors on a spring.

First, the simple cavity controller. Recall from Eq. (33) that the cavity-controller system can be modeled as

$$
\left[\left(\mathrm{Cav}_{2} \boxplus I_{1}\right) \triangleleft(\mathrm{Cav}) \triangleleft\left(\mathrm{Cav}_{1} \boxplus I_{1}\right)\right] \boxplus(\mathrm{Spr})
$$

which has the $S L H$ model

$$
\begin{aligned}
& S=1_{2 \times 2}, \quad L=\left[\sqrt{\kappa_{1}} a, \sqrt{\kappa_{2}} a, \sqrt{\kappa_{m}} b\right], \\
& H=\omega_{c} a^{\dagger} a+\Omega b^{\dagger} b+\sqrt{\kappa_{1}} K_{1} x_{m} p_{c} .
\end{aligned}
$$

Now consider a system, depicted in Fig. 17, consisting of a non-adiabatically-eliminated cavity with one of its mirrors attached to a spring. This has the SLH model

$$
\begin{aligned}
& S=1_{3 \times 3}, \quad L=\left[\sqrt{\kappa_{1}} a, \sqrt{\kappa_{2}} a, \sqrt{k_{m}} b\right], \\
& H=\Delta_{0} a^{\dagger} a+\Omega b^{\dagger} b+\eta a^{\dagger} a x_{m} .
\end{aligned}
$$

A laser $L(\alpha)$ sends a coherent input into mirror 1, giving the system $\operatorname{Cav} \triangleleft\left[L(\alpha) \boxplus I_{2}\right]$. Of course, the internal dynamics do not depend on anything downstream of the system, so we can just as well use $\left[L\left(\alpha^{\prime}\right) \boxplus I_{2}\right] \triangleleft \operatorname{Cav} \triangleleft\left[L(\alpha) \boxplus I_{2}\right]$, for any $\alpha^{\prime}$. Making substitutions $a \rightarrow a-a_{0}, b \rightarrow b-b_{0}$ to center around the equilibrium point, the $S L H$ model becomes

$$
\begin{aligned}
& S=1_{3 \times 3}, \quad L=\left[\sqrt{\kappa_{1}} a, \sqrt{\kappa_{2}} a, \sqrt{k_{m}} b\right] \\
& H=\Delta a^{\dagger} a+\Omega b^{\dagger} b+\frac{\eta|\alpha| \sqrt{\kappa_{1}}}{\Delta^{2}+(\kappa / 2)^{2}} x_{m} x_{c}+\eta a^{\dagger} a x_{m} .
\end{aligned}
$$

Ignoring the nonlinear term, this is almost identical to Eq. (40). One can convert the $x_{m} x_{c}$ term to an $x_{m} p_{c}$ term with a canonical transformation, and the coefficients can be matched by varying $\alpha$. Thus the systems in Eqs. (40) and (42) are equivalent, and the "simple cavity controller" can be realized in the laboratory using a single cavity with a mirror attached to a spring. Cooling an oscillator in this setup has been realized experimentally, though it was not interpreted as a control system [11-13].

The OPO controller is just like the cavity controller, but the Hamiltonian has an additional squeezing term; see (5). The same procedure can be applied to show that the OPO plant-controller system is equivalent to a (non-adiabaticallyeliminated) OPO cavity with a spring mirror, as shown in Fig. 18.

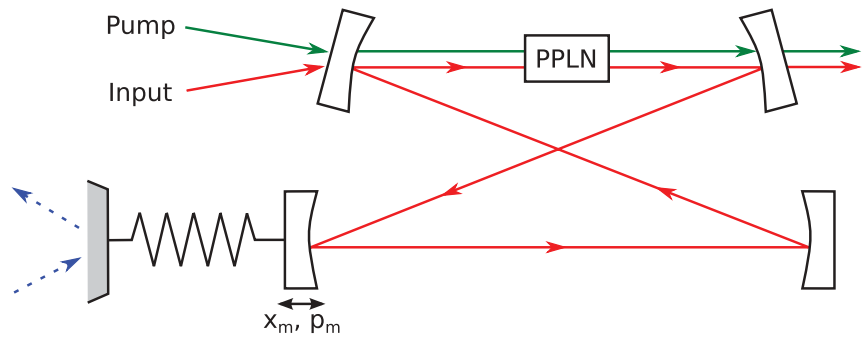

FIG. 18. (Color online) Model for a non-adiabatically-eliminated OPO cavity with a spring mirror.

\section{F. Quantum refrigerator analogy}

One thing we notice from the optimal controller performance is that, in the strong-noise limit, the optimal controllers-classical, OPO, cavity-all reduce the spring phonon number by a factor of about $Q=\Omega / k_{m}$. The classical and OPO controllers reduce it by exactly $Q$, while the cavity controller only reduces it by a factor $Q / 2.83$. This factor-of- $Q$ reduction can be understood by viewing the plant and the controller as thermodynamic systems.

Figure 19 illustrates our point. Starting with a cavity with a spring mirror, we separate the system into the cavity, which oscillates at a frequency $\omega_{c}$, and the spring, which oscillates at a frequency $\Omega$. Each system has its own coupling to the environment. The cavity couples to a vacuum-state environment $(T=0)$ with coupling strengths $\kappa_{1}, \kappa_{2}$, the spring couples to a heat bath with $T_{h}>0$ with strength $k_{m}$, and a spring-cavity coupling $K_{1}=-K_{2}$ couples the two modes.

If the spring and cavity oscillate at about the same frequency and the spring-cavity coupling is strong compared to the other two, then the "temperature" of the spring will be roughly equal to the "temperature" of the cavity. We denote this temperature $T_{\text {sys }}$. One expects the combined system to be in thermal steady state with both the heat bath and vacuum inputs and outputs; this gives us the energy balance equation

$$
k_{m} T_{h}=k_{m} T_{\mathrm{sys}}+\kappa T_{\mathrm{sys}},
$$

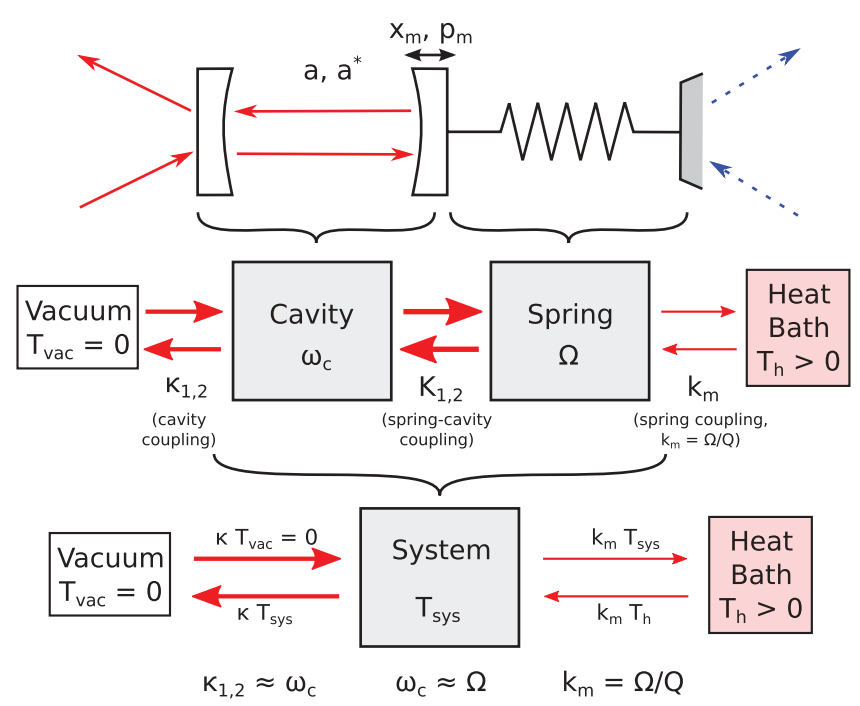

FIG. 19. (Color online) Coherent control problem represented as two coupled thermodynamic systems. 
where $\kappa=\kappa_{1}+\kappa_{2} \sim \omega_{c} \sim \Omega$, and $k_{m}=\Omega / Q$. Solving for the system's steady-state temperature,

$$
T_{\mathrm{sys}}=\frac{k_{m} T_{h}}{\kappa+k_{m}} \sim \frac{T_{h}}{Q} .
$$

From general arguments, we can therefore expect that most good controllers will reduce the spring phonon number by a factor of about $Q$, but that no controller will do significantly better. Note that, since this argument is based on thermodynamic assumptions that are only approximately valid here, the factor-of- $Q$ reduction is only approximate, and only holds in the classical limit. These classical results, unsurprisingly, break down in the quantum regime because, among other things, the effects of vacuum noise inputs become important.

\section{CONCLUSIONS}

In this paper, we have studied the coherent-feedback cooling of linear quantum systems from an LQG control perspective. The systems were modeled using the $S L H$ framework and the Gough-James circuit algebra, which allow arbitrarily large circuits to be constructed in a straightforward and systematic manner. The evolution of the system was studied using QSDEs, the open-system analog to the Heisenberg equations. We wrote MATHEMATICA scripts based on the QHDL framework to model quantum LQG control systems, and designed algorithms to optimize a controller's parameters for a given setup.

For any LQG control problem, there is always a quantum controller that does at least as well as the optimal classical controller. In the quantum regime, when the excitation number in the plant is of order unity, we have shown that the best quantum controller can do better-in some cases, significantly so. Two systems - the optical cavity and the optomechanical oscillator-were studied in detail. For the former, modest gains were found using coherent control in the low-photon-number regime. For the latter, the gains were much larger.

One could imagine extending these results to look at nonquadratic cost functions in linear control systems. Indeed, some work has already been done on this matter, focusing on using coherent feedback to maximize the squeezing in a cavity mode [17]. Taking a control theory perspective may also provide insight into minimizing the noise in optomechanical sensors. In addition, the understanding of the superior performance of coherent feedback in linear systems may provide important clues for the design of quantum controllers for nonlinear systems such as optical switches or error-correcting codes.

\section{ACKNOWLEDGMENTS}

This work has been supported by the NSF (Grant No. PHY-1005386), AFOSR (Grant No. FA9550-11-1-0238), and DARPA-MTO (Grant No. N66001-11-1-4106). R.H. is supported by the NSF GRFP and a Stanford Graduate Fellowship. We thank Nikolas Tezak, Gopal Sarma, Dmitri Pavlichin, and Orion Crisafulli for useful discussions.

\section{APPENDIX A: SLH, QSDE, AND ABCD MODELS}

An open quantum system with $n$ bosonic, Markovian inputoutput channels, can be represented as a triple [28,29]:

$$
(S, L, H)
$$

where $S$ is a unitary $n \times n$ operator-valued matrix, $L$ is an $n$-component operator-valued vector, and $H$ is Hermitian. A quantum circuit is built up by connecting together components of this form. Any such circuit can be written in terms of a circuit algebra of concatenation, series and feedback products, shown in Fig. 20.

The concatenation product joins two systems without connecting any of the input and output ports. If $G_{1}=\left(S_{1}, L_{1}, H_{1}\right)$ and $G_{2}=\left(S_{2}, L_{2}, H_{2}\right)$ then the concatenation is given by

$$
G_{1} \boxplus G_{2}=\left(\left[\begin{array}{ll}
S_{1} & 0 \\
0 & S_{2}
\end{array}\right],\left[\begin{array}{l}
L_{1} \\
L_{2}
\end{array}\right], H_{1}+H_{2}\right) .
$$

The series product feeds the outputs of one system into the inputs of the other, and has the following $S L H$ model:

$$
G_{2} \triangleleft G_{1}=\left(S_{2} S_{1}, L_{2}+S_{2} L_{1}, H_{1}+H_{2}+\operatorname{Im}\left(L_{2}^{\dagger} S_{2} L_{1}\right)\right) \text {. }
$$

The feedback product $[G]_{i \rightarrow j}$ corresponds to taking output $i$ of $G$ and feeding it back into input $j$. This can also be represented with an SLH model. See Ref. [33].

The quantum stochastic differential equations (QSDEs) are Heisenberg-picture equations of motion for open quantum systems. They relate the evolution of the internal state variables (denoted $X$ ) and the output fields $d \tilde{A}_{i}$ and gauge processes $d \tilde{\Lambda}_{i j}$ to the inputs $d A_{i}, d \Lambda_{i j}$, where the inputs are vacuum quantum Wiener processes. For a given $S L H$ model, the equations are [29]

$$
\begin{gathered}
d X=\left[-i[X, H]+\frac{1}{2}\left(L_{i}^{\dagger}\left[X, L_{i}\right]+\left[L_{i}^{\dagger}, X\right] L_{i}\right)\right] d t \\
+d A_{i}^{\dagger} S_{i j}^{\dagger}\left[X, L_{j}\right]+\left[L_{j}^{\dagger}, X\right] S_{j i} d A_{i} \\
+\left(S_{i k}^{\dagger} X S_{k j}-X \delta_{i j}\right) d \Lambda_{i j}, \\
d \tilde{A}_{i}=S_{i j} d A_{j}+L_{i} d t, \\
d \tilde{\Lambda}_{i j}=S_{i k}^{*} d \Lambda_{k l} S_{l j}^{\mathrm{T}}+S_{i k}^{*} d A_{k}^{\dagger} L_{j}+L_{i}^{\dagger} d A_{k} S_{k j}^{\mathrm{T}}+L_{i}^{\dagger} L_{j} .
\end{gathered}
$$

Likewise, the master equation is the Schrödinger-picture equation of motion for an open quantum system. It gives the evolution of the system's density operator:

$$
\frac{d \rho}{d t}=i[\rho, H]+\left(L_{i} \rho L_{i}^{\dagger}-\frac{1}{2} L_{i}^{\dagger} L_{i} \rho-\frac{1}{2} \rho L_{i}^{\dagger} L_{i}\right) .
$$

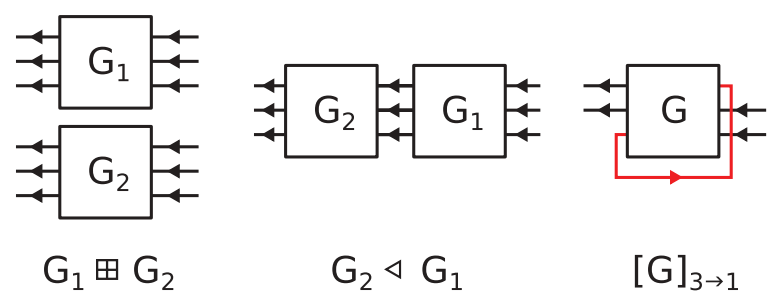

FIG. 20. (Color online) The concatenation, series, and feedback products are the three basic operations of the Gough-James circuit algebra. 
A linear system has a quadratic Hamiltonian and linear environment couplings. It takes the following $S L H$ model:

$$
S_{i j}=S_{i j}, \quad L_{i}=\Lambda_{i} x+\lambda_{i}, \quad H=\frac{1}{2} x^{\mathrm{T}} R x+r^{\mathrm{T}} x .
$$

From these, the $A B C D$ matrices take the forms

$$
\begin{aligned}
A & =2 \Theta\left(R+\frac{1}{4} \tilde{\Lambda}^{\mathrm{T}} J \tilde{\Lambda}\right), \quad B=\Theta \tilde{\Lambda}^{\mathrm{T}} J \tilde{S}, \\
C & =\tilde{\Lambda}, \quad D=\tilde{S} \\
a & =2 \Theta\left(r+\frac{1}{4} \tilde{\Lambda}^{\mathrm{T}} J \tilde{\lambda}\right), \quad c=\tilde{\lambda}, \\
\Theta_{i j} & =\frac{1}{2 i}\left[x_{i}, x_{j}\right] .
\end{aligned}
$$

We form matrices $\tilde{S}$ and $\tilde{\Lambda}$ and vector $\tilde{\lambda}$ by stacking $S, \Lambda$, and $\lambda$ :

$$
\begin{aligned}
\tilde{S}_{a b} & =2 M^{\dagger}\left[\begin{array}{cc}
S_{a b} & 0 \\
0 & S_{a b}^{*}
\end{array}\right] M, \quad \tilde{\Lambda}_{a}=2 M^{\dagger}\left[\begin{array}{c}
\Lambda_{a} \\
\Lambda_{a}^{*}
\end{array}\right], \\
\tilde{\lambda}_{a} & =2 M^{\dagger}\left[\begin{array}{c}
\lambda_{a} \\
\lambda_{a}^{*}
\end{array}\right],
\end{aligned}
$$

where $J_{2 n \times 2 n}$ is the canonical antisymmetric matrix of dimension $2 n$ (written above as $J$, where the dimension is inferred), and $M_{2 n \times 2 n}$ is used to convert between standard $d A_{i}, d A_{i}^{\dagger}$ and Hermitian $d a_{x}, d a_{p}$ input-output fields:

$$
J_{2 n \times 2 n}=I_{n} \otimes\left[\begin{array}{cc}
0 & 1 \\
-1 & 0
\end{array}\right], \quad M_{2 n \times 2 n}=I_{n} \otimes \frac{1}{2}\left[\begin{array}{cc}
1 & i \\
1 & -i
\end{array}\right] .
$$

The matrix $\tilde{S}$ is made from the blocks $\tilde{S}_{a b}$ above, and likewise for $\tilde{\Lambda}$ and $\tilde{\lambda}$.

These match the formulas used in Ref. [18], the difference being that we have defined the $A B C D$ matrices in terms of the real stacked matrices $\tilde{S}$ and $\tilde{\Lambda}$, rather than in terms of $S, L$, and $H$ directly.

\section{APPENDIX B: CIRCUIT ALGEBRA FOR LINEAR MODELS, SLH FORM}

To form the concatenated system $G_{1} \boxplus G_{2}$, we define a new state variable $x=\left[\begin{array}{ll}x_{1} & x_{2}\end{array}\right]$ that includes both the state of $G_{1}$ and the state of $G_{2}$. Applying the concatenation equation (A2),

$$
\begin{aligned}
& S=\left[\begin{array}{ll}
S_{1} & 0 \\
0 & S_{2}
\end{array}\right], \quad \Lambda=\left[\begin{array}{cc}
\Lambda_{1} & 0 \\
0 & \Lambda_{2}
\end{array}\right], \quad R=\left[\begin{array}{l}
R_{1} \\
R_{2}
\end{array}\right], \\
& \lambda=\left[\begin{array}{l}
\lambda_{1} \\
\lambda_{2}
\end{array}\right], \quad r=\left[\begin{array}{l}
r_{1} \\
r_{2}
\end{array}\right] .
\end{aligned}
$$

Likewise, applying the series product equation (A3), one finds the parameters for the system $G_{2} \triangleleft G_{1}$ :

$$
\begin{aligned}
& S=S_{2} S_{1}, \quad \Lambda=\left[\begin{array}{ll}
\Lambda_{1} & \Lambda_{2}
\end{array}\right], \\
& R=\left[\begin{array}{cc}
R_{1} & \operatorname{Im}\left(\Lambda_{2}^{\dagger} S_{2} \Lambda_{1}\right) \\
\operatorname{Im}\left(\Lambda_{2}^{\dagger} S_{2} \Lambda_{1}\right) & R_{2}
\end{array}\right], \\
& \lambda=\lambda_{1}+\lambda_{2}, \quad r=\left[\begin{array}{l}
r_{1}+\operatorname{Im}\left(\lambda_{2}^{\dagger} S_{2} \Lambda_{1}\right)^{\mathrm{T}} \\
r_{2}-\operatorname{Im}\left(\lambda_{2}^{\dagger} S_{2} \Lambda_{1}\right)^{\mathrm{T}}
\end{array}\right] .
\end{aligned}
$$

Similarly, applying the feedback equations can give us the parameters for the system $G^{\prime}=[G]_{i \rightarrow j}$ :

$$
\begin{aligned}
S^{\prime} & =\left[S+S_{* j}\left(1-S_{i j}\right)^{-1} S_{i *}\right]_{! i, ! j}, \\
\Lambda^{\prime} & =\left[\Lambda+S_{* j}\left(1-S_{i j}\right)^{-1} \Lambda_{i *}\right]_{! i, *}, \\
R^{\prime} & =R+\operatorname{Im}\left[\Lambda^{\dagger} S_{* j}\left(1-S_{i j}\right)^{-1} \Lambda-\text { H.c. }\right], \\
\lambda^{\prime} & =\left[\lambda+S_{* j}\left(1-S_{i j}\right)^{-1} \lambda_{i *}\right]_{! i, *}, \\
r^{\prime} & =r+\operatorname{Im}\left[\Lambda^{\dagger} S_{* j}\left(1-S_{i j}\right)^{-1} \lambda+\Lambda^{\mathrm{T}}\left(1-S_{i j}\right)^{-1} S_{j *}^{\mathrm{T}} \lambda^{*}\right],
\end{aligned}
$$

where the notation $*$ means "take all rows (columns) of the given matrix," while ! $j$ means "take all rows (columns) except $j$." For example, $S_{*, j}$ would be the $j$ th column of $S$, while $M_{! i, ! j}$ would be obtained by removing row $i$ and column $j$ from the matrix $M$.

\section{APPENDIX C: CIRCUIT ALGEBRA FOR LINEAR MODELS, ABCD FORM}

Linear quantum systems can also be modeled in terms of their $A, B, C, D, a$, and $c$ matrices. Concatenating two models in $A B C D$ form to create $G_{1} \boxplus G_{2}$ is straightforward:

$$
\begin{aligned}
& A=\left[\begin{array}{cc}
A_{1} & 0 \\
0 & A_{2}
\end{array}\right], \quad B=\left[\begin{array}{cc}
B_{1} & 0 \\
0 & B_{2}
\end{array}\right], \\
& C=\left[\begin{array}{cc}
C_{1} & 0 \\
0 & C_{2}
\end{array}\right], \quad D=\left[\begin{array}{cc}
D_{1} & 0 \\
0 & D_{2}
\end{array}\right], \\
& a=\left[\begin{array}{l}
a_{1} \\
a_{2}
\end{array}\right], \quad c=\left[\begin{array}{l}
c_{1} \\
c_{2}
\end{array}\right] .
\end{aligned}
$$

One can arrive at the series product in $A B C D$ form by first taking the series product in $S L H$ form and then converting to the $A B C D$ matrices. The series product $G=G_{2} \triangleleft G_{1}$ is

$$
\begin{aligned}
A & =\left[\begin{array}{cc}
A_{1} & B_{2} C_{1} \\
0 & A_{2}
\end{array}\right], \quad B=\left[\begin{array}{c}
B_{1} \\
B_{2} D_{1}
\end{array}\right], \\
C & =\left[\begin{array}{ll}
D_{2} C_{1} & C_{2}
\end{array}\right], \quad D=D_{2} D_{1}, \\
a & =\left[\begin{array}{c}
a_{1} \\
a_{2}+B_{2} c_{1}
\end{array}\right], \quad c=c_{2}+D_{2} c_{1} .
\end{aligned}
$$

Likewise, the internal feedback $G^{\prime}=[G]_{i \rightarrow j}$ is given by

$$
\begin{aligned}
A^{\prime} & =A+B_{* j}\left(1-D_{i j}\right)^{-1} C_{i *}, \\
B^{\prime} & =\left[B+B_{* j}\left(1-D_{i j}\right)^{-1} D_{i *}\right]_{*, ! j}, \\
C^{\prime} & =\left[C+D_{* j}\left(1-D_{i j}\right)^{-1} C_{i *}\right]_{! i, *}, \\
D^{\prime} & =\left[D+D_{* j}\left(1-D_{i j}\right)^{-1} D_{i *}\right]_{! i, ! j}, \\
a^{\prime} & =a+B_{* j}\left(1-D_{i j}\right)^{-1} c_{i}, \\
c^{\prime} & =\left[c+D_{* j}\left(1-D_{i j}\right)^{-1} c_{i}\right]_{! i, *} .
\end{aligned}
$$

Alternatively, one can model quantum oscillators using the $A B C D$ models by deriving these relations from a simple inspection of the linear equations of motion, skipping the $S L H$ formalism altogether. This approach is unfavorable because (1) not every $A B C D$ model is a physical system, since the $A B C D$ matrices for a real quantum system must satisfy realizability conditions [19], (2) the $A B C D$ matrices often do not clearly reflect the underlying physics of a device, whereas the Hamiltonian and coupling terms do, and (3) the $A B C D$ 
matrices exist only for linear systems; for future work in the context of nonlinear systems, the $S L H$ formalism will become essential.

\section{APPENDIX D: $S L H$ AND ABCD MODELS FOR SYSTEMS IN THIS PAPER}

As an example, consider three components mentioned in the paper - the empty cavity, the optomechanical oscillator, and the OPO cavity. Start with the empty cavity. This has the $S L H$ model

$$
S=I, \quad L=\left[\sqrt{k_{1}} a, \sqrt{k_{2}} a, \sqrt{k_{3}} a\right], \quad H=\Delta a^{\dagger} a .
$$

Let $x=a+a^{\dagger}, p=\left(a-a^{\dagger}\right) / i$ be the Hermitian state variables for this system. Then we can write out $(S, L, H)$ in the form of Eq. (A8) with the linear system parameters

$$
\begin{aligned}
& S=I_{3 \times 3}, \quad \Lambda=\frac{1}{2}\left[\begin{array}{ll}
\sqrt{k_{1}} & i \sqrt{k_{1}} \\
\sqrt{k_{2}} & i \sqrt{k_{2}} \\
\sqrt{k_{3}} & i \sqrt{k_{3}}
\end{array}\right], \quad R=\frac{1}{2} \Delta I_{2 \times 2}, \\
& r=\lambda=0, \quad \Theta=J_{2 \times 2} .
\end{aligned}
$$

Using Eqs. (A9), the $A B C D$ model is

$$
\begin{aligned}
& A=\left[\begin{array}{cc}
-\frac{k_{1}+k_{2}+k_{3}}{2} & \Delta \\
-\Delta & -\frac{k_{1}+k_{2}+k_{3}}{2}
\end{array}\right], \\
& B=\left[\begin{array}{cccccc}
-\sqrt{k_{1}} & 0 & -\sqrt{k_{2}} & 0 & -\sqrt{k_{2}} & 0 \\
0 & -\sqrt{k_{1}} & 0 & -\sqrt{k_{2}} & 0 & -\sqrt{k_{2}}
\end{array}\right] \text {, } \\
& C=\left[\begin{array}{cc}
\sqrt{k_{1}} & 0 \\
0 & \sqrt{k_{1}} \\
\sqrt{k_{2}} & 0 \\
0 & \sqrt{k_{2}} \\
\sqrt{k_{3}} & 0 \\
0 & \sqrt{k_{3}}
\end{array}\right], \quad D=\left[\begin{array}{cccccc}
1 & 0 & 0 & 0 & 0 & 0 \\
0 & 1 & 0 & 0 & 0 & 0 \\
0 & 0 & 1 & 0 & 0 & 0 \\
0 & 0 & 0 & 1 & 0 & 0 \\
0 & 0 & 0 & 0 & 1 & 0 \\
0 & 0 & 0 & 0 & 0 & 1
\end{array}\right] \text {, } \\
& a=c=0 \text {. }
\end{aligned}
$$

By inspection, one can see that this is equivalent to the wellknown input-output equations for an empty cavity:

$$
\begin{aligned}
d a & =\left(-i \Delta-\frac{1}{2} \sum_{i} k_{i}\right) a d t-\sum_{i} \sqrt{k_{i}} d A_{i}, \\
d \tilde{A}_{i} & =\sqrt{k_{i}} a d t+d A_{i} .
\end{aligned}
$$

In the paper, we also study the control of an optomechanical oscillator. Here, we posited an oscillator with one degree of freedom (the mechanical degree of freedom)

$$
S=1_{2 \times 2}, \quad L=\left[K x_{m}, \sqrt{\Omega / Q} b\right], \quad H=\Omega b^{\dagger} b,
$$

where $\left(x_{m}, p_{m}\right)$ are the Hermitian state variables and $b=\left(x_{m}+\right.$ $\left.i p_{m}\right) / 2$ is the phonon annihilation operator. Again referring to Eq. (A8), the linear system parameters are

$$
\begin{aligned}
& S=I_{2 \times 2}, \quad \Lambda=\left[\begin{array}{cc}
K & 0 \\
\frac{1}{2} \sqrt{\Omega / Q} & \frac{i}{2} \sqrt{\Omega / Q}
\end{array}\right], \quad R=\frac{\Omega}{2} I_{2 \times 2}, \\
& r=\lambda=0, \quad \Theta=J_{2 \times 2} .
\end{aligned}
$$

Again, following the standard procedure, we derive $A B C D$ matrices for the model:

$$
\begin{aligned}
& A=\Omega\left[\begin{array}{cc}
-1 / 2 Q & 1 \\
-1 & -1 / 2 Q
\end{array}\right], \\
& B=\left[\begin{array}{cccc}
0 & 0 & -\sqrt{\Omega / Q} & 0 \\
0 & -2 K & 0 & -\sqrt{\Omega / Q}
\end{array}\right] \text {, } \\
& C=\left[\begin{array}{cc}
2 K & 0 \\
0 & 0 \\
\sqrt{\Omega / Q} & 0 \\
0 & \sqrt{\Omega / Q}
\end{array}\right], \quad D=\left[\begin{array}{llll}
1 & 0 & 0 & 0 \\
0 & 1 & 0 & 0 \\
0 & 0 & 1 & 0 \\
0 & 0 & 0 & 1
\end{array}\right] \text {. }
\end{aligned}
$$

This model is consistent with the equations of motion

$$
\begin{aligned}
& d x_{m}=\Omega p_{m}-\Omega / 2 Q x_{m}-\sqrt{\Omega / Q} d a_{2 x}, \\
& d p_{m}=-\Omega x_{m}-\Omega / 2 Q p_{m}-2 K d a_{1 p}-\sqrt{\Omega / Q} d a_{2 p}, \quad \text { (D8) } \\
& d \tilde{a}_{1 x}=d a_{1 x}+2 K x_{m} d t, \quad d \tilde{a}_{1 p}=d a_{1 p}, \\
& d \tilde{a}_{2 x}=d a_{2 x}+\sqrt{\Omega / Q} x_{m} d t, \quad d \tilde{a}_{2 p}=d a_{2 p}+\sqrt{\Omega / Q} p_{m} d t,
\end{aligned}
$$

which were stated without proof in the paper. (For clarity, the phonon mode $d a_{2 x}, d a_{2 p}$ was omitted in the paper.)

Finally, we consider the OPO cavity. Although not studied as a plant, the OPO has interesting properties as a controller for the mechanical oscillator system. The OPO has the following SLH model:

$$
\begin{aligned}
& S=1_{2 \times 2}, \quad L=\left[\sqrt{\kappa_{1}} a, \quad \sqrt{\kappa_{2}} a\right], \\
& H=\Delta a^{\dagger} a+\frac{\epsilon^{*} a^{2}-\epsilon\left(a^{\dagger}\right)^{2}}{2 i} .
\end{aligned}
$$

Once more referring to Eq. (A8) and turning the crank, the linear system parameters are

$$
\begin{aligned}
& S=1_{2 \times 2}, \quad \Lambda=\frac{1}{2}\left[\begin{array}{cc}
\sqrt{\kappa_{1}} & i \sqrt{\kappa_{1}} \\
\sqrt{\kappa_{2}} & i \sqrt{\kappa_{2}}
\end{array}\right], \\
& R=\frac{1}{2}\left[\begin{array}{cc}
\Delta-\operatorname{Im}(\epsilon) & \operatorname{Re}(\epsilon) \\
\operatorname{Re}(\epsilon) & \Delta+\operatorname{Im}(\epsilon)
\end{array}\right], \\
& r=\lambda=0, \quad \Theta=J_{2 \times 2},
\end{aligned}
$$

and the $A B C D$ matrices are

$$
\begin{aligned}
& A=\left[\begin{array}{cc}
\operatorname{Re}(\epsilon)-\frac{\kappa_{1}+\kappa_{2}}{2} & \Delta+\operatorname{Im}(\epsilon) \\
-\Delta+\operatorname{Im}(\epsilon) & -\operatorname{Re}(\epsilon)-\frac{\kappa_{1}+\kappa_{2}}{2}
\end{array}\right], \\
& B=\left[\begin{array}{cccc}
-\sqrt{\kappa_{1}} & 0 & -\sqrt{\kappa_{2}} & 0 \\
0 & -\sqrt{\kappa_{1}} & 0 & -\sqrt{\kappa_{2}}
\end{array}\right] \text {, } \\
& C=\left[\begin{array}{cc}
\sqrt{\kappa_{1}} & 0 \\
0 & \sqrt{\kappa_{1}} \\
\sqrt{\kappa_{2}} & 0 \\
0 & \sqrt{\kappa_{2}}
\end{array}\right], \quad D=\left[\begin{array}{cccc}
1 & 0 & 0 & 0 \\
0 & 1 & 0 & 0 \\
0 & 0 & 1 & 0 \\
0 & 0 & 0 & 1
\end{array}\right] \text {. }
\end{aligned}
$$


[1] K. Åstrom and R. M. Murray, Feedback Systems: An Introduction for Scientists and Engineers (Princeton University Press, Princeton, NJ, 2008).

[2] V. P. Belavkin, Autom. Remote Control 44, 178 (1983).

[3] H. M. Wiseman and G. J. Milburn, Phys. Rev. Lett. 70, 548 (1993).

[4] A. C. Doherty, S. Habib, K. Jacobs, H. Mabuchi, and S. M. Tan, Phys. Rev. A 62, 012105 (2000).

[5] H. Mabuchi and N. Khaneja, Int. J. Robust Nonlinear Control 15, 647 (2005)

[6] D. Dong and I. R. Petersen, IET Control Theory Applic. 4, 2651 (2010).

[7] C. Brif, R. Chakrabarti, and H. Rabitz, New J. Phys. 12, 075008 (2010).

[8] W. P. Smith, J. E. Reiner, L. A. Orozco, S. Kuhr, and H. M. Wiseman, Phys. Rev. Lett. 89, 133601 (2002).

[9] M. A. Armen, J. K. Au, J. K. Stockton, A. C. Doherty, and H. Mabuchi, Phys. Rev. Lett. 89, 133602 (2002).

[10] P. Bushev, D. Rotter, A. Wilson, F. Dubin, C. Becher, J. Eschner, R. Blatt, V. Steixner, P. Rabl, and P. Zoller, Phys. Rev. Lett. 96, 043003 (2006).

[11] S. Gigan, H. R. Böhm, M. Paternostro, F. Blaser, G. Langer, J. B. Hertzberg, K. C. Schwab, D. Bäuerle, M. Aspelmeyer, and A. Zeilinger, Nature (London) 444, 67 (2006).

[12] O. Arcizet, P.-F. Cohadon, T. Briant, M. Pinard, and A. Heidmann, Nature (London) 444, 71 (2006).

[13] D. Kleckner and D. Bouwmeester, Nature (London) 444, 75 (2006).

[14] H. Mabuchi, Phys. Rev. A 78, 032323 (2008).

[15] G. G. Gillett, R. B. Dalton, B. P. Lanyon, M. P. Almeida, M. Barbieri, G. J. Pryde, J. L. O’Brien, K. J. Resch, S. D. Bartlett, and A. G. White, Phys. Rev. Lett. 104, 080503 (2010).

[16] C. Sayrin, I. Dotsenko, X. Zhou, B. Peaudecerf, T. Rybarczyk, S. Gleyzes, P. Rouchon, M. Mirrahimi, H. Amini, M. Brune, J.-M. Raimond, and S. Haroche, Nature (London) 477, 73 (2011).

[17] S. Iida, M. Yukawa, H. Yonezawa, N. Yamamoto, and A. Furusawa, IEEE Trans. Autom. Control 57, 2045 (2012).

[18] M. R. James, H. I. Nurdin, and I. R. Petersen, IEEE Trans. Autom. Control 53, 1787 (2008).

[19] H. I. Nurdin, M. R. James, and I. R. Petersen, Automatica 45, 1847 (2009).

[20] R. L. Hudson and K. R. Parthasarathy, Commun. Math. Phys. 93, 301 (1984).

[21] H. J. Carmichael, Phys. Rev. Lett. 70, 2273 (1993).

[22] C. W. Gardiner, Phys. Rev. Lett. 70, 2269 (1993).
[23] A. Barchielli, in Open Quantum Systems III: Recent Developments, edited by S. Attal, A. Joye, and C.-A. Pillet (Springer, Berlin, 2006).

[24] J. Kerckhoff, H. I. Nurdin, D. S. Pavlichin, and H. Mabuchi, Phys. Rev. Lett. 105, 040502 (2010).

[25] J. Kerckhoff, D. S. Pavlichin, H. Chalabi, and H. Mabuchi, New J. Phys. 13, 055022 (2011).

[26] H. Mabuchi, Appl. Phys. Lett. 98, 193109 (2011).

[27] J. E. Gough, M. R. James, H. I. Nurdin, and J. Combes, Phys. Rev. A 86, 043819 (2012).

[28] J. Gough and M. R. James, Commun. Math. Phys. 287, 1109 (2009).

[29] J. Gough and M. R. James, IEEE Trans. Autom. Control 54, 2530 (2009).

[30] C. W. Gardiner and P. Zoller, Quantum Noise: A Handbook of Markovian and Non-Markovian Quantum Stochastic Methods with Applications to Quantum Optics (Springer, Berlin, 2004).

[31] L. Bouten, R. Van Handel, and M. R. James, SIAM J. Control Optim. 46, 2199 (2007).

[32] G. Sarma, R. Hamerly, N. Tezak, D. S. Pavlichin, and H. Mabuchi, arXiv:1206.1104.

[33] N. Tezak, A. Niederberger, D. Pavlichin, G. Sarma, and H. Mabuchi, arXiv:1111.3081 [Philos. Trans. R. Soc., A (to be published)].

[34] H. I. Nurdin, M. R. James, and A. C. Doherty, SIAM J. Control Optim. 48, 2686 (2009).

[35] D. Simon, Optimal State Estimation: Kalman, $H^{\infty}$, and Nonlinear Approaches (Wiley-Interscience, New York, 2006).

[36] L. Bouten and A. Silverfarb, Commun. Math. Phys. 283, 491 (2008).

[37] L. Bouten, R. Van Handel, and A. Silverfarb, J. Funct. Anal. 254, 3123 (2008).

[38] J. E. Gough, H. I. Nurdin, and S. Wildfeuer, J. Math. Phys. 51, 123518 (2010).

[39] F. Marquardt and S. Girvin, Physics 2, 40 (2009).

[40] T. Botter, D. W. C. Brooks, N. Brahms, S. Schreppler, and D. M. Stamper-Kurn, Phys. Rev. A 85, 013812 (2012).

[41] H. Miao, S. Danilishin, H. Müller-Ebhardt, and Y. Chen, New J. Phys. 12, 083032 (2010).

[42] F. Marquardt, J. P. Chen, A. A. Clerk, and S. M. Girvin, Phys. Rev. Lett. 99, 093902 (2007).

[43] I. Wilson-Rae, N. Nooshi, W. Zwerger, and T. J. Kippenberg, Phys. Rev. Lett. 99, 093901 (2007).

[44] M. Tsang and C. M. Caves, Phys. Rev. Lett. 105, 123601 (2010).

[45] O. Arcizet, T. Briant, A. Heidmann, and M. Pinard, Phys. Rev. A 73, 033819 (2006). 